\title{
ANALYTIC PROPERTIES OF MIRROR MAPS
}

\author{
C. KRATTENTHALER and T. RIVOAL ${ }^{凶}$
}

(Received 2 April 2011; accepted 16 January 2012)

Communicated by I. E. Shparlinski

Dedicated to the memory of Alf van der Poorten

\begin{abstract}
We consider a multi-parameter family of canonical coordinates and mirror maps originally introduced by Zudilin. This family includes many of the known one-variable mirror maps as special cases, in particular many of modular origin and the celebrated 'quintic' example of Candelas, de la Ossa, Green and Parkes. In a previous paper, we proved that all coefficients in the Taylor expansions at 0 of these canonical coordinates (and, hence, of the corresponding mirror maps) are integers. Here we prove that all coefficients in their Taylor expansions at 0 are positive. Furthermore, we provide several results about the behaviour of the canonical coordinates and mirror maps as complex functions. In particular, we address their analytic continuation, points of singularity, and radius of covergence. We present several very precise conjectures on the radius of covergence of the mirror maps and the sign pattern of the coefficients in their Taylor expansions at 0 .
\end{abstract}

2010 Mathematics subject classification: primary 30B10; secondary 11F11, 14J32, 30B40, 33C20.

Keywords and phrases: mirror maps, canonical coordinates, analytic continuation, singular expansion, generalised hypergeometric functions, modular forms.

\section{Introduction}

This paper focuses on a multi-parameter family of canonical coordinates and mirror maps originally introduced by Zudilin [39] (to be defined below). This family contains many of the known one-variable mirror maps as special cases, including many of modular origin and the celebrated example of Candelas et al. [10] associated to the quintic hypersurface in $\mathbb{P}^{4}(\mathbb{C})$. For the geometric significance of these maps; see $[10,26,27,36]$. The number-theoretic properties of the coefficients in the Taylor

The research of the first author was partially supported by the Austrian Science Foundation FWF, grants Z130-N13 and S9607-N13, the latter in the framework of the National Research Network 'Analytic Combinatorics and Probabilistic Number Theory'. The research of the second author was partially supported by the project Q-DIFF, ANR-10-JCJC-0105, of the French 'Agence Nationale de la Recherche'.

(C) 2012 Australian Mathematical Publishing Association Inc. 1446-7887/2012 \$16.00 
expansions at 0 of these canonical coordinates and mirror maps have been investigated recently in [20] (see [13] for a far-reaching generalisation). Our aim here is to provide as detailed an analysis as possible of the analytic properties of canonical coordinates and mirror maps. Apart from the intrinsic interest in this kind of investigation, one motivation comes from the hope of finding more applications of the Diophantine method of uniformisation adélique simultanée of André [2], notably to nonmodular situations.

Another connection between number theory and mirror maps can be found in the study of the arithmetic nature of values of the Riemann zeta function at integers. Apéry's proof of the irrationality of $\zeta(3)$ (see [30]) was recast in terms of modular forms by Beukers [7]. The search for an extension of Beukers' ideas to $\zeta(n)$ when $n \geq 4$ led Almkvist and Zudilin [1] to systematically study mirror maps associated to Fuchsian differential equations, not necessarily of hypergeometric type. In [21], the authors showed that, in fact, many of the examples of Beukers and of Almkvist and Zudilin can be obtained from suitable specialisations of hypergeometric multivariable mirror maps. It would therefore be of great interest to extend the investigations undertaken in this paper addressing the analytic behaviour of the family of mirror maps to be introduced below to the family of multi-variable mirror maps in [21].

Let us now introduce this family of canonical coordinates and corresponding mirror maps. Given a positive integer $N$, denote by $r_{1}, r_{2}, \ldots, r_{d}$ the integers in $\{1,2, \ldots, N\}$ that are coprime to $N$. It is well known that $d=\varphi(N)$, where $\varphi$ is Euler's totient function, given by $\varphi(N)=N \prod_{p \mid N}\left(1-p^{-1}\right)$. Set $C_{N}:=N^{\varphi(N)} \prod_{p \mid N} p^{\varphi(N) /(p-1)}$, which is an integer because $p-1$ divides $\varphi(N)$ for any prime $p$ dividing $N$. Let us also define the Pochhammer symbol $(\alpha)_{m}$ for complex numbers $\alpha$ and nonnegative integers $m$ by $(\alpha)_{m}:=\alpha(\alpha+1) \cdots(\alpha+m-1)$ if $m \geq 1$, and $(\alpha)_{0}:=1$. It can be proved (see [39, Lemma 1]) that $\mathbf{B}_{N}(m)$ is an integer for any nonnegative integer $m$, where

$$
\mathbf{B}_{N}(m):=C_{N}^{m} \prod_{j=1}^{\varphi(N)} \frac{\left(r_{j} / N\right)_{m}}{m !} .
$$

Let us consider the hypergeometric differential operator $\mathbf{L}$ defined by

$$
\mathbf{L}:=\left(z \frac{d}{d z}\right)^{\varphi\left(N_{1}\right)+\cdots+\varphi\left(N_{k}\right)}-C_{\mathbf{N}} z \prod_{j=1}^{k} \prod_{i=1}^{\varphi\left(N_{j}\right)}\left(z \frac{d}{d z}+\frac{r_{i, j}}{N_{j}}\right) .
$$

Here, $C_{\mathbf{N}}=C_{N_{1}} C_{N_{2}} \cdots C_{N_{k}}$ and the $r_{i, j} \in\left\{1,2, \ldots, N_{j}\right\}$ form the residue classes modulo $N_{j}$ which are coprime to $N_{j}$. Unless $k=1$ and $\mathbf{N}=(2)$, the differential equation $\mathbf{L} y=0$ is of order two or more. We can construct two solutions as follows. Set $H(x, m):=\sum_{n=0}^{m-1}(x+n)^{-1}$ and

$$
\mathbf{H}_{N}(m):=\sum_{j=1}^{\varphi(N)} H\left(r_{j} / N, m\right)-\varphi(N) H(1, m) .
$$


Then, $\mathbf{F}_{\mathbf{N}}(z)$ and $\mathbf{G}_{\mathbf{N}}(z)+\log (z) \mathbf{F}_{\mathbf{N}}(z)$ are two $\mathbb{C}$-linearly independent solutions to $\mathbf{L} y=0$, where

$$
\mathbf{F}_{\mathbf{N}}(z):=\sum_{m=0}^{\infty}\left(\prod_{j=1}^{k} \mathbf{B}_{N_{j}}(m)\right) z^{m}
$$

and

$$
\mathbf{G}_{\mathbf{N}}(z):=\sum_{m=1}^{\infty}\left(\sum_{j=1}^{k} \mathbf{H}_{N_{j}}(m)\right)\left(\prod_{j=1}^{k} \mathbf{B}_{N_{j}}(m)\right) z^{m},
$$

and where log denotes the principal branch of the logarithm. For simplicity, we write $\mathbf{B}_{\mathbf{N}}(m):=\prod_{j=1}^{k} \mathbf{B}_{N_{j}}(m)$ and $\mathbf{H}_{\mathbf{N}}(m):=\sum_{j=1}^{k} \mathbf{H}_{N_{j}}(m)$. Since $\mathbf{B}_{1}(m)=1$ and $\mathbf{H}_{1}(m)=0$ whenever $m \geq 0$, the series $\mathbf{F}_{\mathbf{N}}(z)$ and $\mathbf{G}_{\mathbf{N}}(z)$ do not change if components of 1 are omitted from or added to $\mathbf{N}$. We may therefore assume without loss of generality that $N_{j} \geq 2$ for all $j$, and we shall do so throughout the paper.

The power series $\mathbf{F}_{\mathbf{N}}(z)$ and $\mathbf{G}_{\mathbf{N}}(z)$ have radius of convergence $1 / C_{\mathbf{N}}$. We prove in Section 3 that the functions $\mathbf{F}_{\mathbf{N}}(z)$ and $\mathbf{G}_{\mathbf{N}}(z)+\log (z) \mathbf{F}_{\mathbf{N}}(z)$ can be analytically continued to $\mathbb{C} \backslash\left[1 / C_{\mathbf{N}},+\infty\right)$ and $\mathbb{C} \backslash\left((-\infty, 0] \cup\left[1 / C_{\mathbf{N}},+\infty\right)\right)$.

Given the notation above, we define the canonical coordinate $\mathbf{q}_{\mathbf{N}}(z)$ as the exponential of the quotient of the above two solutions, that is, by

$$
\mathbf{q}_{\mathbf{N}}(z):=z \exp \left(\mathbf{G}_{\mathbf{N}}(z) / \mathbf{F}_{\mathbf{N}}(z)\right)
$$

Its compositional inverse, which we denote by $\mathbf{z}_{\mathbf{N}}(q)$, is called (the corresponding) mirror map.

When $k=1$ and $\mathbf{N}=(2)$, we have $\mathbf{F}_{(2)}(z)=(1-4 z)^{-1 / 2}$, which satisfies the differential equation $(1-4 z) y^{\prime}-2 y=0$. The function $\mathbf{G}_{(2)}(z)+\log (z) \mathbf{F}_{(2)}(z)$ defined formally by the above formula is not a solution of that differential equation, but it turns out that all theorems stated below are still true in this case because

$$
\mathbf{q}_{(2)}(z)=(1-\sqrt{1-4 z})^{2} /(4 z) .
$$

However, certain proofs do not work for this case, and we will say when.

The special case where $\mathbf{N}=(5)$ has been of particular interest since it produces the earlier mentioned example of Candelas et al. [10].

In [20], we proved that, for any positive integers $N_{1}, N_{2}, \ldots, N_{k}$, the canonical coordinate $\mathbf{q}_{\mathbf{N}}(z)$ has integral Taylor coefficients. Our first result says that these coefficients are, in fact, positive. Its proof is given in Section 2. An essential ingredient there is a classical result of Kaluza [19] on the sign of coefficients in certain power series expansions (see Lemma 2.2).

Theorem 1.1. For all integers $N_{1}, N_{2}, \ldots, N_{k}$, each of which is at least 2, all Taylor coefficients of $\mathbf{q}_{\mathbf{N}}(z)$ at 0 are positive, except the constant coefficient, which vanishes.

A problem that suggests itself at this point is to find a combinatorial interpretation for the Taylor coefficients of $\mathbf{q}_{\mathbf{N}}(z)$ or of $\mathbf{z}_{\mathbf{N}}(q)$ (even if the latter may have negative 
coefficients; see Conjecture 1.9 below). Some progress in this direction can be found in [23].

The next theorem provides precise information on the radius of convergence and the asymptotic behaviour of the Taylor coefficients of the canonical coordinate $\mathbf{q}_{\mathbf{N}}(z)$ as a power series in $z$. Henceforth, given $\mathbf{N}=\left(N_{1}, N_{2}, \ldots, N_{k}\right)$, we employ the notation

$$
\Phi_{\mathbf{N}}:=\sum_{j=1}^{k} \varphi\left(N_{j}\right) .
$$

THeOREM 1.2. For all integers $N_{1}, N_{2}, \ldots, N_{k}$, each of which is at least 2 , the following assertions hold.

(i) The radius of convergence of the Taylor series of $\mathbf{q}_{\mathbf{N}}(z)$ is equal to $1 / C_{\mathbf{N}}$ and the Taylor series converges for any $z$ such that $|z|=1 / C_{\mathbf{N}}$.

(ii) The function $\mathbf{q}_{\mathbf{N}}(z)$ has a singularity at $z=1 / C_{\mathbf{N}}$.

(iii) For any $z$ such that $|z| \leq 1 / C_{\mathbf{N}}$, we have $\left|\mathbf{q}_{\mathbf{N}}(z)\right| \leq 1$.

(iv) If $\Phi_{\mathbf{N}}=1$ and $m \geq 1$, then the mth Taylor coefficient of $\mathbf{q}_{(2)}(z)$ is equal to the mth Catalan number $(m+1)^{-1}\left(\begin{array}{c}2 m \\ m\end{array}\right)$, and hence as $m \rightarrow \infty$, it is equal to

$$
\frac{4^{m}}{\sqrt{\pi} m^{3 / 2}}(1+o(1)) .
$$

(v) If $\Phi_{\mathbf{N}}=2$, then, as $m$ tends to $\infty$, the mth Taylor coefficient of $\mathbf{q}_{\mathbf{N}}(z)$ is equal to

$$
K \frac{C_{\mathbf{N}}^{m}}{m \log ^{2}(m)}(1+o(1)),
$$

where $K$ is a nonzero constant.

(vi) If $\Phi_{\mathbf{N}} \geq 3$, then, as $m$ tends to $\infty$, the mth Taylor coefficient of $\mathbf{q}_{\mathbf{N}}(z)$ is equal to

$$
K \frac{C_{\mathbf{N}}^{m}}{m^{\Phi_{\mathbf{N}} / 2}}(1+o(1)),
$$

where $K$ is a nonzero constant.

In (iii), the inequality is always strict, except at $z=1 / C_{\mathbf{N}}$ when the series $\mathbf{F}_{\mathbf{N}}\left(1 / C_{\mathbf{N}}\right)$ diverges to $+\infty$, which happens in five cases: $k=1, N_{1}=2,3,4$ or 6 , and $k=2$, $N_{1}=N_{2}=2$.

Theorem 1.2 is proved in Section 5. There, the analytic continuation of $\mathbf{F}_{\mathbf{N}}(z)$ and $\mathbf{G}_{\mathbf{N}}(z)$, which is discussed in Section 3, plays an important role, as well as the fine behaviour of these functions near the point $1 / C_{\mathbf{N}}$, which is discussed in detail in Section 4.

By (1.3), the canonical coordinate $\mathbf{q}_{\mathbf{N}}(z)$ is only defined for $z$ in the disk of convergence of the series $\mathbf{G}_{\mathbf{N}}(z)$ and $\mathbf{F}_{\mathbf{N}}(z)$ involved in its definition. The knowledge of the analytic continuation of $\mathbf{F}_{\mathbf{N}}(z)$ and $\mathbf{G}_{\mathbf{N}}(z)$ from Section 3, combined with a theorem of Pólya [29] on zeros of hypergeometric functions, allows us to show that $\mathbf{q}_{\mathbf{N}}(z)$ can be continued to a function of the entire complex plane except for a branch cut. The corresponding proof is the subject of Section 6. 
THeOREM 1.3. For all integers $N_{1}, N_{2}, \ldots, N_{k}$, each of which is at least 2 , the following assertions hold.

(i) The power series $\mathbf{q}_{\mathbf{N}}(z)$ can be continued analytically to $\mathbb{C} \backslash\left[1 / C_{\mathbf{N}},+\infty\right)$.

(ii) The point $1 / C_{\mathbf{N}}$ is a branch point.

(iii) For $M_{\mathbf{N}}=\max \left(N_{1}, \ldots, N_{k}\right)$,

$$
\lim _{z \rightarrow \infty} \mathbf{q}_{\mathbf{N}}(z)=-\exp \left(-\pi \cot \left(\pi / M_{\mathbf{N}}\right)\right)
$$

where the limit has to be taken along a path that avoids the cut $\left[1 / C_{\mathbf{N}},+\infty\right)$.

The monodromy of $\mathbf{q}_{\mathbf{N}}(z)$ at $z=1 / C_{\mathbf{N}}$ follows from applying the well-known monodromy theory of solutions to (generalised) hypergeometric equations (see [8]) to the series $\mathbf{F}_{\mathbf{N}}(z)$ and $\mathbf{G}_{\mathbf{N}}(z)+\log (z) \mathbf{F}_{\mathbf{N}}(z)$.

The function $\mathbf{q}_{\mathbf{N}}(z)$, seen as a formal power series in $z$, is invertible at $z=0$. Its formal inverse $\mathbf{z}_{\mathbf{N}}(q)$ is a power series in $q$ which converges in a neighbourhood of $q=0$ (see [17, Theorems $2.4 \mathrm{~b}, \mathrm{c}]$ ). The corresponding results for this compositional inverse $\mathbf{z}_{\mathbf{N}}(q)$ that we are able to establish are less complete than Theorems 1.1-1.3 for $\mathbf{q}_{\mathbf{N}}(z)$. First of all, we use Theorem 1.3 to get some information on the radius of convergence of $\mathbf{z}_{\mathbf{N}}(q)$ as a power series in $q$.

THEOREM 1.4. Let $N_{1}, N_{2}, \ldots, N_{k}$ be integers, all at least 2 .

(i) The radius of convergence of the Taylor series of $\mathbf{z}_{\mathbf{N}}(q)$ is at most $\exp \left(-\pi \cot \left(\pi / M_{\mathbf{N}}\right)\right)$.

(ii) If $\Phi_{\mathbf{N}} \geq 4$, then the radius of convergence of the Taylor series of $\mathbf{z}_{\mathbf{N}}(q)$ is at most $\mathbf{q}_{\mathbf{N}}\left(1 / C_{\mathbf{N}}\right)$.

The proof of this theorem is found in Section 7.

Lemma 8.1 in Section 8 provides a comparison of the two values that feature in the above theorem in the case where $\Phi_{\mathbf{N}} \geq 4$ : namely, there it is shown that $\exp \left(-\pi \cot \left(\pi / M_{\mathbf{N}}\right)\right)>\mathbf{q}_{\mathbf{N}}\left(1 / C_{\mathbf{N}}\right)$. As we shall see in Section 9 , this inequality continues to hold when $\Phi_{\mathbf{N}}=3$, but it is wrong for the remaining cases when $\Phi_{\mathbf{N}}=1,2$.

In the cases of 'small' $\Phi_{\mathbf{N}}$, we are able to provide precise information on the analytic continuation of $\mathbf{z}_{\mathbf{N}}(q)$; see the theorem below. As we explain in Section 9, this information is in fact essentially available in the literature on the modular origin of the corresponding mirror maps. We point out that there exist several other hypergeometric functions (not covered by our series $\mathbf{F}_{\mathbf{N}}(z)$ ) which give rise to mirror maps of modular origin; see, for instance, [35, 40]. It would be of interest to know whether there is an analogous result for these mirror maps.

Theorem 1.5. For all integers $N_{1}, N_{2}, \ldots, N_{k}$, each of which is at least 2, such that $\Phi_{\mathbf{N}} \leq 3$, the radius of convergence of the Taylor series of $\mathbf{z}_{\mathbf{N}}(q)$ is equal to $\exp \left(-\pi \cot \left(\pi / M_{\mathbf{N}}\right)\right)$. The function $\mathbf{z}_{\mathbf{N}}(q)$ can be analytically continued to the unit disk with the exception of a set of poles or branch points which can be described precisely. In particular, $-\exp \left(-\pi \cot \left(\pi / M_{\mathbf{N}}\right)\right)$ is always a singularity of $\mathbf{z}_{\mathbf{N}}(q)$. If $\mathbf{N}=(2)$, we 


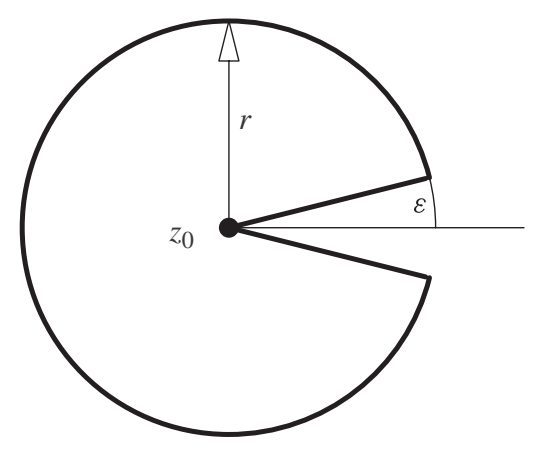

FIGURE 1 . The right slit neighbourhood $\Delta\left(z_{0} ; r, \varepsilon\right)$ of $z_{0}$.

have $\mathbf{z}_{\mathbf{N}}(q)=q /(1+q)^{2}$. In all the eight other cases, the unit circle forms a natural boundary for $\mathbf{z}_{\mathbf{N}}(q)$.

In Section 9, we also find precise information on the poles and branch points.

In the case where $\Phi_{\mathbf{N}} \geq 4$, we can offer only the partial result below on the analytic nature of $\mathbf{z}_{\mathbf{N}}(q)$; the proof is given in Section 10. There, and later, we use the notion of a right slit neighbourhood of $z_{0}$, by which we mean a domain of the form

$$
\Delta\left(z_{0} ; r, \varepsilon\right):=\left\{z:\left|z-z_{0}\right|<r \text { and }\left|\arg \left(z-z_{0}\right)\right|>\varepsilon\right\}
$$

for some $r, \varepsilon>0$, where it is understood that $\arg \left(z-z_{0}\right)$ is taken from $(-\pi, \pi]$; see Figure 1. A left slit neighbourhood is defined in the obvious analogous way.

Proposition 1.6. For all integers $N_{1}, N_{2}, \ldots, N_{k}$, each of which is at least 2 , such that $\Phi_{\mathbf{N}} \geq 4$, the series $\mathbf{z}_{\mathbf{N}}(q)$ can be analytically continued to a domain that contains the half-open segment $\left[0, \mathbf{q}_{\mathbf{N}}\left(1 / C_{\mathbf{N}}\right)\right)$. Moreover, this domain can be chosen so that it also contains a right slit neighbourhood of $\mathbf{q}_{\mathbf{N}}\left(1 / C_{\mathbf{N}}\right)$, where the angle of the slit, controlled by the parameter $\varepsilon$ in (1.7), can be chosen arbitrarily small.

Remark 1.7. The function $\mathbf{q}_{\mathbf{N}}(z)$ can also be locally inverted at $z=\infty$. Consequently, the corresponding local inverse function $\tilde{\mathbf{z}}_{\mathbf{N}}(q)$ is defined in a left slit neighbourhood of $-\exp \left(-\pi \cot \left(\pi / M_{\mathbf{N}}\right)\right.$ ) (see the last paragraph in Section 10). The point $-\exp \left(-\pi \cot \left(\pi / M_{\mathbf{N}}\right)\right)$ is always a singular point. Whether it is a pole, a branch point, or an essential singularity can be decided by inspecting the expansions at $\infty$ for $\mathbf{q}_{\mathbf{N}}(z)$ given in Lemma 4.1 (taking also into consideration the remark after the statement of the lemma), and by subsequently applying Lemma 4.7, with the accompanying remark in mind. Unfortunately, we do not know how to relate $\tilde{\mathbf{z}}_{\mathbf{N}}(q)$ and $\mathbf{z}_{\mathbf{N}}(q)$.

Certainly, the above result cannot even answer the innocent question of what the radius of convergence of $\mathbf{z}_{\mathbf{N}}(q)$ as a power series in $q$ is. However, we performed extensive calculations using the computer algebra system PARI/GP [28], in which we computed approximations up to many digits to the radius of convergence using the 
quotient rule. These computations provide abundant evidence for the exact value of the radius of convergence for the cases where $\Phi_{\mathbf{N}}$ is 'large'.

Conjecture 1.8. For all integers $N_{1}, N_{2}, \ldots, N_{k}$, each of which is at least 2 , such that $\Phi_{\mathbf{N}} \geq 4$, the radius of convergence of the Taylor series of $\mathbf{z}_{\mathbf{N}}(q)$ is equal to $\mathbf{q}_{\mathbf{N}}\left(1 / C_{\mathbf{N}}\right)$. Moreover, the point $\mathbf{q}_{\mathbf{N}}\left(1 / C_{\mathbf{N}}\right)$ is the only singularity of $\mathbf{z}_{\mathbf{N}}(q)$ on the boundary of its disk of convergence.

We discuss a possible line of argument to prove this conjecture in Section 11.

When $\Phi_{\mathbf{N}}=2,3$, Theorem 1.5 shows that the mirror map $\mathbf{z}_{\mathbf{N}}(q)$ has a natural boundary, but our computer experiments for $\Phi_{\mathbf{N}} \geq 4$ did not allow us to develop an intuition whether or not there is a natural boundary for $\mathbf{z}_{\mathbf{N}}(q)$ in these cases.

Concerning the Taylor coefficients of $\mathbf{z}_{\mathbf{N}}(q)$ at $q=0$, our numerical calculations suggest very predictable behaviour.

Conjecture 1.9. Let $N_{1}, N_{2}, \ldots, N_{k}$ be positive integers, all at least 2.

(i) If $\Phi_{\mathbf{N}} \leq 3$, the Taylor coefficients of $\mathbf{z}_{\mathbf{N}}(q)$ have alternating signs.

(ii) If $\Phi_{\mathbf{N}}=4$, the coefficients of $q$ and of $q^{3}$ in the Taylor series of $\mathbf{z}_{\mathbf{N}}(q)$ are positive, while all other coefficients are negative, except the constant coefficient, which vanishes.

(iii) If $\Phi_{\mathbf{N}} \geq 5$, the coefficient of $q$ in the Taylor series of $\mathbf{z}_{\mathbf{N}}(q)$ is positive, while all other coefficients are negative, except the constant coefficient, which vanishes.

We show in Section 12 that Conjecture 1.9 implies the first assertion in Conjecture 1.8. On the other hand, as we show in Section 13, Conjecture 1.8 implies a weak version of Conjecture 1.9 .

There are three cases where Conjecture 1.9(i) is a theorem. In the case where $\mathbf{N}=$ $(2,6)$, where $\mathbf{z}_{(2,6)}(q)=1 / j(\tau)$ (in which $q=\exp (2 i \pi \tau)$ and $j(\tau)$ is the Dedekind-Klein $j$-invariant; see Section 9.3), this has been established by Asai et al. [4, Corollary 2, p. 94]. In the case where $\mathbf{N}=(2,2)$, it follows immediately that the coefficients alternate upon inspection of the explicit expression for $\mathbf{z}_{(2,2)}(q)$ given in (9.8). Similarly, in the case where $\mathbf{N}=(2,2,2)$, the alternating sign of the coefficients follows immediately upon inspection of the explicit expression for $\mathbf{z}_{(2,2,2)}(q)$ given in (9.14). Moreover, Theorem 1.5, Lemmas 4.3 and 4.7, together with the standard theorems of singularity analysis (see [14, Ch. VI]), imply that Conjecture 1.9(i) holds asymptotically, in the sense that the coefficients of $q^{m}$ of $\mathbf{z}_{\mathbf{N}}(q)$ alternate in sign for all sufficiently large $m$.

The expression for $\mathbf{q}_{\mathbf{N}}(z)$ in terms of $\mathbf{F}_{\mathbf{N}}(z)$ and $\mathbf{G}_{\mathbf{N}}(z)$ is not very convenient for a study of the fine analytic properties of $\mathbf{q}_{\mathbf{N}}(z)$ and of the corresponding mirror map $\mathbf{z}_{\mathbf{N}}(q)$. The following conjecture, extending Theorem 1.1, is motivated by the search for an alternative expression, as we explain below.

Conjecture 1.10. For all integers $N_{1}, N_{2}, \ldots, N_{k}$, each of which is at least 2, and positive integers $n$, the coefficient of $z^{m}$ in $\left(C_{\mathbf{N}} z-1\right)^{n} \mathbf{q}_{\mathbf{N}}(z)$ is positive whenever $m \geq n+1$. 
Computer calculations indicate that the coefficients of $z^{m}$ in $\left(C_{\mathbf{N}} z-1\right)^{n} \mathbf{q}_{\mathbf{N}}(z)$ are actually alternating when $m \leq n+1$ until the sign stabilises as described in the conjecture. That it must stabilise eventually follows from the singular expansion for $\mathbf{q}_{\mathbf{N}}(z)$ given in Lemma 4.3 together with the standard theorems of singularity analysis (see [14, Ch. VI]). So the point here is that the sign stabilises already for $m \geq n+1$.

By a classical theorem of Hausdorff [16], Conjecture 1.10 implies that the sequence of coefficients of $\mathbf{q}_{\mathbf{N}}(z)$ is a moment sequence for a finite measure $d \chi(t)$ on $\left(0, C_{\mathbf{N}}\right)$. As a consequence, $\mathbf{q}_{\mathbf{N}}(z)$ could be written in the form

$$
\mathbf{q}_{\mathbf{N}}(z)=z \int_{0}^{C_{\mathbf{N}}} \frac{d \chi(t)}{1-z t} .
$$

By a theorem of Wirths [37], it would then follow that $\mathbf{q}_{\mathbf{N}}(z)$ is univalent in the open half plane $\operatorname{Re}(z)<1 / C_{\mathbf{N}}$. In particular, this would lead to the following strengthening of Proposition 1.6: for all integers $N_{1}, N_{2}, \ldots, N_{k}$, each of which is at least 2, such that $\Phi_{\mathbf{N}} \geq 4$, the series $\mathbf{z}_{\mathbf{N}}(q)$ can be analytically continued to a domain that contains the open segment $\left(-\exp \left(-\pi \cot \left(\pi / M_{\mathbf{N}}\right)\right), \mathbf{q}_{\mathbf{N}}\left(1 / C_{\mathbf{N}}\right)\right)$. Moreover, this domain can be chosen so that it contains a right slit neighbourhood of $\mathbf{q}_{\mathbf{N}}\left(1 / C_{\mathbf{N}}\right)$ and a left slit neighbourhood of $-\exp \left(-\pi \cot \left(\pi / M_{\mathrm{N}}\right)\right.$ ) (where the angle of the slit can be chosen arbitrarily small; see the last paragraph in Section 10).

From now on, to simplify notation, we let $C:=C_{\mathrm{N}}, \Phi:=\Phi_{\mathrm{N}}$, and $M:=M_{\mathrm{N}}$.

\section{Proof of Theorem 1.1}

A formal power series $f(z)=\sum_{n=0}^{\infty} f_{n} z^{n} \in \mathbb{C}[[z]]$ with $f_{0} \neq 0$ is invertible in $\mathbb{C}[[z]]$. We can thus see that $\widehat{f}(z)=1-1 / f(z)$ as a formal power series. For the proof of Theorem 1.1, we need the following auxiliary result, whose proof can be found later in this section.

Lemma 2.1. Let $f(z)=\sum_{n=0}^{\infty} f_{n} z^{n}$, where $f_{0}=1$, be such that the Taylor coefficients of $\widehat{f}(z)$ are nonnegative. Consider $g(z)=\sum_{n=0}^{\infty} h_{n} f_{n} z^{n}$ where the sequence of real numbers $\left(h_{n}\right)_{n \geq 0}$ is nondecreasing and nonnegative. Then the Taylor coefficients of $g(z) / f(z)$ are nonnegative.

If, in addition, all Taylor coefficients of $f$ and $\widehat{f}$ are positive (except the constant coefficient of $\widehat{f})$ and the sequence $\left(h_{n}\right)_{n \geq 0}$ is strictly increasing, then the Taylor coefficients of $g(z) / f(z)$ are positive, except the constant coefficient if $h_{0}=0$.

To apply Lemma 2.1 to our situation, we need two further results. The first is a theorem due to Kaluza [19, Satz 3]. Strictly speaking, the assertion in [19, Satz 3] deals only with the condition $f_{n+1} f_{n-1} \geq f_{n}^{2}$; however, the strengthening given below is easily extracted from the proof in [19].

Lemma 2.2. Let $f(z)=\sum_{n=0}^{\infty} f_{n} z^{n}, f_{0}=1$, be such that $f_{1}>0$ and $f_{n+1} f_{n-1} \geq f_{n}^{2}$ whenever $n \geq 1$. Then the Taylor coefficients of $\widehat{f}(z)$ are nonnegative. 
If the stronger condition $f_{n+1} f_{n-1}>f_{n}^{2}$ is satisfied for all positive integers $n$, then the Taylor coefficients of $\widehat{f}(z)$ are positive (except the constant coefficient).

(It is easy to see that, if $\widehat{f}(z)$ has nonnegative Taylor coefficients, then $f_{n} \geq 0$ whenever $n \geq 0$, but that the converse is not true in general.)

Lemma 2.3. Fix the integers $N_{1}, N_{2}, \ldots, N_{k}$, each of which is at least 2. Then $\mathbf{B}_{\mathbf{N}}(1)>0$ and

$$
\mathbf{B}_{\mathbf{N}}(m+1) \mathbf{B}_{\mathbf{N}}(m-1)>\mathbf{B}_{\mathbf{N}}(m)^{2}
$$

whenever $m \geq 1$. Furthermore, the sequence $\left(\mathbf{H}_{\mathbf{N}}(m)\right)_{m \geq 0}$ is positive and strictly increasing.

The proof of this lemma can be found at the end of this section. The combination of Lemmas 2.2 and 2.3 immediately implies the following corollary.

Corollary 2.4. Let $N_{1}, N_{2}, \ldots, N_{k}$ be positive integers, all at least 2. Then the Taylor coefficients of $\widehat{\mathbf{F}}_{\mathbf{N}}(z)$ are positive (except the constant coefficient).

The above corollary shows that we can take $f=\mathbf{F}_{\mathbf{N}}$ and $g=\mathbf{G}_{\mathbf{N}}$ in Lemma 2.1. Hence, the power series $\mathbf{G}_{\mathbf{N}}(z) / \mathbf{F}_{\mathbf{N}}(z)$ has positive Taylor coefficients (except the constant coefficient), a property which obviously remains true when we take the exponential. This proves Theorem 1.1.

Proof of Lemma 2.1. Let us write $\widehat{f}(z)=\sum_{n=1}^{\infty} \widehat{f}_{n} z^{n}$. The relation $f(z)(1-\widehat{f}(z))=1$ translates to

$$
f_{n}-\sum_{k=1}^{n} \widehat{f}_{k} f_{n-k}=\delta_{n, 0} \quad \text { for all } n \geq 0
$$

where $\delta_{n, 0}$ is the Kronecker symbol. Furthermore,

$$
\frac{g(z)}{f(z)}=g(z)(1-\widehat{f}(z))=\sum_{n=0}^{\infty} z^{n}\left(h_{n} f_{n}-\sum_{k=1}^{n} \widehat{f_{k}} f_{n-k} h_{n-k}\right) .
$$

Since $\left(h_{n}\right)_{n \geq 0}$ is nondecreasing and nonnegative, by (2.1),

$$
h_{n} f_{n}-\sum_{k=1}^{n} \widehat{f}_{k} f_{n-k} h_{n-k} \geq h_{n} f_{n}-\sum_{k=1}^{n} \widehat{f_{k}} f_{n-k} h_{n}=h_{n}\left(f_{n}-\sum_{k=1}^{n} \widehat{f}_{k} f_{n-k}\right)=h_{n} \delta_{n, 0} \geq 0 .
$$

The additional assertion in the lemma follows as well from the above arguments by observing that, because of the stronger assumptions, the first inequality in (2.2) is strict when $n \geq 1$.

Proof of Lemma 2.3 It is clear that $\mathbf{B}_{\mathbf{N}}(m)>0$ whenever $m \geq 0$.

We want to prove that

$$
\frac{\mathbf{B}_{\mathbf{N}}(m+1) \mathbf{B}_{\mathbf{N}}(m-1)}{\mathbf{B}_{\mathbf{N}}(m)^{2}}>1
$$


whenever $m \geq 1$. By definition,

$$
\frac{\mathbf{B}_{\mathbf{N}}(m+1) \mathbf{B}_{\mathbf{N}}(m-1)}{\mathbf{B}_{\mathbf{N}}(m)^{2}}=\prod_{j=1}^{k} \prod_{i=1}^{\varphi\left(N_{j}\right)} \frac{\left(r_{i, j} / N\right)_{m+1}\left(r_{i, j} / N\right)_{m-1}}{\left(r_{i, j} / N\right)_{m}^{2}} .
$$

We observe that for every positive real number $x$ and every positive integer $m$,

$$
\frac{(x)_{m+1}(x)_{m-1}}{(x)_{m}^{2}}=\frac{x+m}{x+m-1}>1,
$$

which immediately implies (2.3).

Concerning the second claim,

$$
\begin{aligned}
\mathbf{H}_{\mathbf{N}}(m) & =\sum_{j=1}^{k}\left(\sum_{i=1}^{\varphi\left(N_{j}\right)} H\left(r_{i, j} / N, m\right)-\varphi\left(N_{j}\right) H(1, m)\right) \\
& =\sum_{j=1}^{k} \sum_{i=1}^{\varphi\left(N_{j}\right)}\left(H\left(r_{i, j} / N_{j}, m\right)-H(1, m)\right),
\end{aligned}
$$

from which we deduce that

$$
\mathbf{H}_{\mathbf{N}}(m+1)-\mathbf{H}_{\mathbf{N}}(m)=\sum_{j=1}^{k} \sum_{i=1}^{\varphi\left(N_{j}\right)}\left(\frac{1}{m+r_{i, j} / N_{j}}-\frac{1}{m+1}\right)>0,
$$

because $0<r_{i, j} / N_{j}<1$. Since $\mathbf{H}_{\mathbf{N}}(0)=0$, we have proved that the sequence $\left(\mathbf{H}_{\mathbf{N}}(m)\right)_{m \geq 1}$ is positive and strictly increasing.

\section{Analytic continuation of $\mathbf{F}_{\mathrm{N}}(z)$ and $\mathbf{G}_{\mathrm{N}}(z)$}

For $z$ complex with $|\arg (-z)|<\pi$, let $\log (z)$ denote the branch of the logarithm which assigns values with imaginary part between 0 and $2 \pi$. We shall continue to use $\log$ for the principal branch of the logarithm. In order to facilitate the reading of the following paragraphs, as a rule of thumb, below, whenever $z^{\gamma}$ appears, it has to be understood as $z^{\gamma}=\exp (\gamma \log (z))$, whereas whenever $(-z)^{\gamma}$ appears, it has to be understood as $(-z)^{\gamma}=\exp (\gamma \log (-z))$.

For a nonnegative real number $h$, set

$$
F(h, z):=\sum_{n=0}^{\infty} \frac{\left(\alpha_{1}+h\right)_{n} \cdots\left(\alpha_{\Phi}+h\right)_{n}}{(1+h)_{n}^{\Phi}} z^{n+h},
$$

where $\Phi=\Phi_{\mathbf{N}}$ is given by (1.5) and the $\alpha_{j}$ run through the elements of the multiset (a multiset is a 'set' where one allows repetitions of elements)

$$
\left\{r_{i, j} / N_{j}: i=1, \ldots, \varphi\left(N_{j}\right), j=1, \ldots, k\right\} .
$$


We have

$$
F(0, C z)=\mathbf{F}_{\mathbf{N}}(z) \quad \text { and } \quad \frac{\partial F}{\partial h}(0, C z)=\log (C z) \mathbf{F}_{\mathbf{N}}(z)+\mathbf{G}_{\mathbf{N}}(z)
$$

Generalised hypergeometric functions, such as the sum on the right-hand side of (3.1), have a Barnes-type integral representation; see [34, Section 4.6]. If we apply this to the right-hand side of (3.1) then, for any complex number $z$ such that $|\arg (-z)|<\pi$ and any $h \geq 0$, we obtain

$$
\begin{aligned}
\sum_{n=0}^{\infty} & \frac{\left(\alpha_{1}+h\right)_{n} \cdots\left(\alpha_{\Phi}+h\right)_{n}}{(1+h)_{n}^{\Phi}} z^{n} \\
=- & \frac{1}{2 i \pi} \frac{\Gamma(1+h)^{\Phi}}{\Gamma\left(\alpha_{1}+h\right) \cdots \Gamma\left(\alpha_{\Phi}+h\right)} \\
& \times \int_{\mathscr{C}} \frac{\Gamma\left(\alpha_{1}+h+s\right) \cdots \Gamma\left(\alpha_{\Phi}+h+s\right)}{\Gamma(1+h+s)^{\Phi}} \frac{\pi}{\sin (\pi s)}(-z)^{s} d s,
\end{aligned}
$$

where $\mathscr{C}$ is a path from $-i \infty$ to $+i \infty$ such that $0,1,2, \ldots$ lie on the right of $\mathscr{C}$ and the poles of the $\Gamma\left(\alpha_{\ell}+h+s\right), \ell=1, \ldots, \Phi$, lie to the left.

By multiplying both sides of (3.3) by $z^{h}$, and by using the relation $\log (z)=$ $\log (-z)+i \pi$ (recall the convention on the branches of the logarithm in the first paragraph of this section), we obtain, when $|\arg (-z)|<\pi$ and $h \geq 0$, the equation

$$
\begin{aligned}
F(h, z)=- & \frac{e^{i \pi h}}{2 i \pi} \frac{\Gamma(1+h)^{\Phi}}{\Gamma\left(\alpha_{1}+h\right) \cdots \Gamma\left(\alpha_{\Phi}+h\right)} \\
& \times \int_{\mathscr{C}} \frac{\Gamma\left(\alpha_{1}+h+s\right) \cdots \Gamma\left(\alpha_{\Phi}+h+s\right)}{\Gamma(1+h+s)^{\Phi}} \frac{\pi}{\sin (\pi s)}(-z)^{s+h} d s .
\end{aligned}
$$

In particular, when $h=0$ and $z$ is changed to $C z$, this provides the analytic continuation of $\mathbf{F}_{\mathbf{N}}(z)$ to the cut plane $|\arg (-z)|<\pi$. Since $\mathbf{F}_{\mathbf{N}}(z)$ is also analytic at any real point $z \in\left[0,1 / C\right.$, we get in this way the analytic continuation of $\mathbf{F}_{\mathbf{N}}(z)$ to the cut plane $|\arg (1 / C-z)|<\pi$.

We now differentiate both sides of (3.4) with respect to $h$, and then set $h=0$ and change $z$ to $C z$. After simplification, we obtain

$$
\begin{aligned}
\mathbf{G}_{\mathbf{N}}(z)= & \left(\Phi \psi(1)-\sum_{\ell=1}^{\Phi} \psi\left(\alpha_{\ell}\right)\right) \mathbf{F}_{\mathbf{N}}(z) \\
& -\frac{1}{2 i \pi \Gamma\left(\alpha_{1}\right) \cdots \Gamma\left(\alpha_{\Phi}\right)} \int_{\mathscr{C}} \frac{\Gamma\left(\alpha_{1}+s\right) \cdots \Gamma\left(\alpha_{\Phi}+s\right)}{\Gamma(1+s)^{\Phi}} \\
& \times\left(\sum_{\ell=1}^{\Phi} \psi\left(\alpha_{\ell}+s\right)-\Phi \psi(1+s)\right) \frac{\pi(-C z)^{s}}{\sin (\pi s)} d s,
\end{aligned}
$$

where $\psi$ is the digamma function. A standard argument shows that the integral on the right-hand side of (3.5) is analytic in the cut plane $|\arg (-z)|<\pi$, hence this is also the 
case for $\mathbf{G}_{\mathbf{N}}(z)$. Then, from the series representation (1.2), we conclude that, in fact, $\mathbf{G}_{\mathbf{N}}(z)$ is analytic in the cut plane $|\arg (1 / C-z)|<\pi$.

In [20, p. 216], we proved that

$$
\left(\Phi \psi(1)-\sum_{\ell=1}^{\Phi} \psi\left(\alpha_{\ell}\right)\right)=\sum_{j=1}^{k} \sum_{i=1}^{\varphi\left(N_{j}\right)}\left(\psi(1)-\psi\left(r_{i, j} / N\right)\right)=\log (C) .
$$

This quantity will reappear later.

We now prove a result that will be needed in the proof of Theorem 1.3(iii) in Section 6. Here, $\mathbf{F}_{\mathbf{N}}(z)$ and $\mathbf{G}_{\mathbf{N}}(z)$ are given by their analytic continuations discussed just above, while $\log (z)$ denotes the branch of the logarithm described at the beginning of this section.

Lemma 3.1. For all integers $N_{1}, N_{2}, \ldots, N_{k}$, each of which is at least 2, let $M=$ $\max \left(N_{1}, \ldots, N_{k}\right)$, as before. Then

$$
\lim _{z \rightarrow \infty}\left(\log (z)+\frac{\mathbf{G}_{\mathbf{N}}(z)}{\mathbf{F}_{\mathbf{N}}(z)}\right)=-\pi \cot (\pi / M)+i \pi,
$$

where the limit has to be taken along a path that avoids the cut $[0,+\infty)$.

Proof. By a well-known method, the integral (3.4) enables us to obtain an alternative expression for $F(h, z)$ when $|z|>1 / C$ in the cut plane $|\arg (1 / C-z)|<\pi$ : we shift the contour $\mathscr{C}$ to the left, taking into account the various poles of the integrand coming from the product $\Gamma\left(\alpha_{1}+s\right) \cdots \Gamma\left(\alpha_{\Phi}+s\right)$.

Let us start with the case where $k=1$, in which case $\alpha_{j}=r_{j} / N$. Then, when $|z|>1 / C$ and $|\arg (1 / C-z)|<\pi$,

$$
\begin{aligned}
F(h, z)=e^{i \pi h} & \sum_{j=1}^{\varphi(N)} \frac{\pi \Gamma(1+h)^{\varphi(N)}}{\sin \left(\pi\left(\frac{r_{j}}{N}+h\right)\right) \prod_{\ell=1}^{\varphi(N)} \Gamma\left(\frac{r_{\ell}}{N}+h\right)} \\
& \times \sum_{\ell=0}^{\infty} \frac{\prod_{\ell=1, \ell \neq j}^{\varphi(N)} \Gamma\left(\frac{r_{\ell}-r_{j}}{N}-\ell\right)}{\ell ! \Gamma\left(1-\frac{r_{j}}{N}-\ell\right)^{\varphi(N)}}(-z)^{-\ell-r_{j} / N},
\end{aligned}
$$

and similarly

$$
\begin{aligned}
\frac{\partial F}{\partial h}(h, z)=\sum_{j=1}^{\varphi(N)} & \frac{\partial}{\partial h}\left(e^{i \pi h} \frac{\pi \Gamma(1+h)^{\varphi(N)}}{\sin \left(\pi\left(\frac{r_{j}}{N}+h\right)\right) \prod_{\ell=1}^{\varphi(N)} \Gamma\left(\frac{r_{\ell}}{N}+h\right)}\right) \\
& \times \sum_{\ell=0}^{\infty} \frac{\prod_{\ell=1, \ell \neq j}^{\varphi(N)} \Gamma\left(\frac{r_{\ell}-r_{j}}{N}-\ell\right)}{\ell ! \Gamma\left(1-\frac{r_{j}}{N}-\ell\right)^{\varphi(N)}}(-z)^{-\ell-r_{j} / N} .
\end{aligned}
$$

In both cases, the leading term is the one corresponding to $(-z)^{-1 / N}$, and thus

$$
\lim _{z \rightarrow \infty} \frac{\frac{\partial F}{\partial h}(0, z)}{F(0, z)}=\log (C)-\pi \cot (\pi / N)+i \pi
$$


(Again, we use (3.6) to get the value $\log (C)$.) Using (3.2), the lemma follows in this case.

In the general case, it can be much more complicated to compute precisely the expansions because the poles might have multiplicity (that is, some of the $\alpha_{j}$ might be equal or differ by an integer). The expected expansions are linear forms in the functions

$$
(-z)^{-\alpha_{\ell}} \log ^{j}(-z) g_{\ell, j}(1 / z), \quad j=0, \ldots, \beta_{\ell}-1,
$$

with coefficients that depend on $h$. Here $\beta_{\ell}$ is the multiplicity of $\alpha_{\ell}$, and the $g_{\ell, j}(z)$ are holomorphic functions at $z=0$. The main terms in the expansions of $\mathbf{F}_{\mathbf{N}}(z)$ and $\mathbf{G}_{\mathbf{N}}(z)+\log (C z) \mathbf{F}_{\mathbf{N}}(z)$ are those corresponding to $(-z)^{-1 / M} \log ^{\beta-1}(-z)$ (with the same maximal $\beta$ in both cases), which can be computed without difficulty from (3.4). We again get

$$
\lim _{z \rightarrow \infty} \frac{\frac{\partial F}{\partial h}(0, z)}{F(0, z)}=\log (C)-\pi \cot (\pi / M)+i \pi,
$$

and the lemma follows.

\section{Singular expansions for $\mathrm{q}_{\mathrm{N}}(z)$ and $\mathrm{z}_{\mathrm{N}}(q)$}

The purpose of this section is to discuss the singular expansions of $\mathbf{q}_{\mathbf{N}}(z)$ at $z=\infty$ (see Lemma 4.1) and $z=1 / C_{\mathbf{N}}$ (see Lemma 4.3), and of $\mathbf{z}_{\mathbf{N}}(q)$ at the corresponding points $q=-\exp \left(-\pi \cot \left(\pi / M_{\mathbf{N}}\right)\right)$ (recall (1.6)) and $q=\mathbf{q}_{\mathbf{N}}\left(1 / C_{\mathbf{N}}\right)$. To obtain the latter, we combine Lemma 4.1 with Lemmas 4.4-4.6, or Lemma 4.3 with Lemma 4.7.

We start with the singular expansion of $\mathbf{q}_{\mathbf{N}}(z)$ at $z=\infty$.

Lemma 4.1. Let $N_{1}, N_{2}, \ldots, N_{k}$ be positive integers, all of which are at least 2 , and let $M=M_{\mathbf{N}}$, as before. Furthermore, let $R$ be the smallest number different from $1 / M$ in the multiset

$$
\Re=\left\{r_{i, j} / N_{j}: i=1,2, \ldots, \varphi\left(N_{j}\right), j=1,2, \ldots, k\right\} .
$$

(i) If both $1 / M$ and $R$ appear exactly once in the multiset $\Re$, then $\mathbf{q}_{\mathbf{N}}(z)$ admits a singular expansion at $\infty$ of the form

$$
\mathbf{q}_{\mathbf{N}}(z)=\mathfrak{q}_{0}+\mathfrak{q}_{1}(-z)^{-R+1 / M}+O\left((-z)^{-R+1 / M-1 / L} \log ^{B_{3}-1}(-z)\right),
$$

where $\mathfrak{q}_{0}=-\exp (-\pi \cot (\pi / M)), \mathfrak{q}_{1}$ is a nonzero constant, $L=\operatorname{lcm}\left(N_{1}, N_{2}\right.$, $\left.\ldots, N_{k}\right)$, and $B_{3}$ is the multiplicity of the third smallest element in $\Re$.

(ii) If $1 / M$ appears exactly once in the multiset $\Re$, while $R$ appears with multiplicity $B_{2}$, then $\mathbf{q}_{\mathbf{N}}(z)$ admits a singular expansion at $\infty$ of the form

$$
\mathbf{q}_{\mathbf{N}}(z)=\mathfrak{q}_{0}+\mathfrak{q}_{1}(-z)^{-R+1 / M} \log ^{B_{2}-1}(-z)+O\left((-z)^{-R+1 / M} \log ^{B_{2}-2}(-z)\right),
$$

where $\mathfrak{q}_{0}$ and $\mathfrak{q}_{1}$ are the same constants as in (i). 
(iii) If $1 / M$ appears with multiplicity at least 2 in $\Re$, then $\mathbf{q}_{\mathbf{N}}(z)$ admits a singular expansion at $\infty$ of the form

$$
\mathbf{q}_{\mathbf{N}}(z)=\mathfrak{q}_{0}+\widetilde{\mathfrak{q}}_{1} \log ^{-1}(-z)+O\left(\log ^{-2}(-z)\right),
$$

where $\mathfrak{q}_{0}$ has the same meaning as in (i), and $\widetilde{\mathfrak{q}}_{1}$ is a nonzero constant.

REMARK 4.2. If the multiset $\Re$ is in fact a set, that is, if all elements of $\mathfrak{R}$ appear with multiplicity 1 , then $\mathbf{q}_{\mathbf{N}}(z)$ admits a Puiseux expansion in $(-z)^{-1 / L}$, of which (4.1) shows the first terms. In all other cases, the singular expansion at $z=\infty$ has terms containing $\log (-z)$.

Proof of Lemma 4.1. (i) By (3.2) and (if $B_{3} \geq 2$, the appropriately generalised) expansions (3.7) and (3.8), we know that

$$
\mathbf{q}_{\mathbf{N}}(z)=\frac{1}{C} \exp \left(\frac{\frac{\partial F}{\partial h}(0, C z)}{F(0, C z)}\right)
$$

where

$$
F(0, C z)=F_{1}(-C z)^{-1 / M}+F_{2}(-C z)^{-R}+O\left((-z)^{-R-1 / L} \log ^{B_{3}-1}(-z)\right)
$$

and

$$
\frac{\partial F}{\partial h}(0, C z)=G_{1}(-C z)^{-1 / M}+G_{2}(-C z)^{-R}+O\left((-z)^{-R-1 / L} \log ^{B_{3}-1}(-z)\right) .
$$

Here, $F_{1}, F_{2}, G_{1}, G_{2}$ are explicit nonzero constants. If we use this in (4.4), then we obtain

$$
\mathbf{q}_{\mathbf{N}}(z)=\frac{1}{C} \exp \left(\frac{G_{1}}{F_{1}}\left(1+\left(\frac{G_{2}}{G_{1}}-\frac{F_{2}}{F_{1}}\right)(-C z)^{-R+1 / M}+O\left((-z)^{-R+1 / M-1 / L} \log ^{B_{3}-1}(-z)\right)\right)\right) .
$$

From the explicit expressions and (3.6), it is not difficult to see that $G_{1} / F_{1}=\log (C)-$ $\pi \cot (\pi / M)+i \pi$ and that, furthermore, $G_{2} / G_{1} \neq F_{2} / F_{1}$. The assertions in (i) now follow easily upon expansion of the exponential.

(ii) We proceed in the same way as in (i). Here, the expansions (4.5) and (4.6) must be replaced by

$$
F(0, C z)=F_{1}(-C z)^{-1 / M}+F_{2}(-C z)^{-R} \log ^{B_{2}-1}(-z)+O\left((-z)^{-R} \log ^{B_{2}-2}(-z)\right)
$$

and

$$
\frac{\partial F}{\partial h}(0, C z)=G_{1}(-C z)^{-1 / M}+G_{2}(-C z)^{-R} \log ^{B_{2}-1}(-z)+O\left((-z)^{-R} \log ^{B_{2}-2}(-z)\right) .
$$

The constants $F_{1}, F_{2}, G_{1}$ and $G_{2}$ are the same as in (i). The remaining steps leading to (4.2) are completely analogous to those in (i) and are therefore omitted. 
(iii) Again, we proceed in the same way as in (i). Here, the expansions (4.5) and (4.6) must be replaced by

$$
\begin{gathered}
F(0, C z)=F_{1}(-C z)^{-1 / M} \log ^{B_{1}-1}(-z)+\widetilde{F}_{2}(-C z)^{-1 / M} \log ^{B_{1}-2}(-z) \\
+O\left((-z)^{-1 / M} \log ^{B_{2}-3}(-z)\right)
\end{gathered}
$$

and

$$
\begin{aligned}
\frac{\partial F}{\partial h}(0, C z)=G_{1}(-C z)^{-1 / M} \log ^{B_{1}-1}(-z)+\widetilde{G}_{2}(-C z)^{-1 / M} \log ^{B_{1}-2}(-z) \\
+O\left((-z)^{-1 / M} \log ^{B_{1}-3}(-z)\right)
\end{aligned}
$$

The constants $F_{1}$ and $F_{2}$ are the same as in (i). The remaining steps leading to (4.3) are completely analogous to those in (i) and are therefore omitted.

The next lemma addresses the singular expansion of $\mathbf{q}_{\mathbf{N}}(z)$ at $z=1 / C_{\mathbf{N}}$.

Lemma 4.3. Let $N_{1}, N_{2}, \ldots, N_{k}$ be positive integers, all of which are at least 2 , and let $C=C_{\mathbf{N}}, \Phi=\Phi_{\mathbf{N}}$, as before.

(i) If $\Phi=2$, then $\mathbf{q}_{\mathbf{N}}(z)$ admits a singular expansion at $1 / C$ of the form

$$
\mathbf{q}_{\mathbf{N}}(z)=1+\mathbf{q}_{1} \log ^{-1}(1-C z)+O\left(\log ^{-2}(1-C z)\right),
$$

where $\mathrm{q}_{1}>0$.

(ii) If $\Phi \geq 3$ is odd, then $\mathbf{q}_{\mathbf{N}}(z)$ admits a singular expansion at $1 / C$ of the form

$$
\begin{aligned}
\mathbf{q}_{\mathbf{N}}(z)=\mathbf{q}_{\mathbf{N}}(1 / C)+\mathrm{q}_{1}(1-C z)+\mathrm{q}_{2}(1-C z)^{2}+\cdots \\
\quad+\mathrm{q}_{d}(1-C z)^{d}+\mathrm{q}_{d+1 / 2}(1-C z)^{d+1 / 2}+O\left((1-C z)^{d+1}\right),
\end{aligned}
$$

where $d=\frac{1}{2}(\Phi-3), \mathrm{q}_{1}<0$, and $(-1)^{d+1} \mathrm{q}_{d+1 / 2}>0$.

(iii) If $\Phi \geq 4$ is even, then $\mathbf{q}_{\mathbf{N}}(z)$ admits a singular expansion at $1 / C$ of the form

$$
\begin{aligned}
\mathbf{q}_{\mathbf{N}}(z)=\mathbf{q}_{\mathbf{N}} & (1 / C)+\mathrm{q}_{1}(1-C z)+\mathrm{q}_{2}(1-C z)^{2}+\cdots \\
& +\mathrm{q}_{d-1}(1-C z)^{d-1}+\mathrm{q}_{d+}(1-C z)^{d} \log (1-C z) \\
& +O\left((1-C z)^{d}\right),
\end{aligned}
$$

where $d=\frac{1}{2}(\Phi-2), \mathrm{q}_{1}<0$, and $(-1)^{d+1} \mathrm{q}_{d+}>0$.

Proof. We proceed by using the theory of hypergeometric differential equations to determine the form of the singular expansion of the quotient $\mathbf{G}_{\mathbf{N}}(z) / \mathbf{F}_{\mathbf{N}}(z)$; see (4.19). This is then translated in the final step into the claimed singular expansions for $\mathbf{q}_{\mathbf{N}}(z)$.

First of all, from Section 3 we know that $\mathbf{F}_{\mathbf{N}}(z)$ and $\mathbf{G}_{\mathbf{N}}(z)+\log (a z) \mathbf{F}_{\mathbf{N}}(z)$ (for any $a \neq 0)$ can be analytically continued to $\mathbb{C} \backslash[1 / C,+\infty)$ and $\mathbb{C} \backslash((-\infty, 0] \cup[1 / C,+\infty))$. We want to determine their behaviour around the point $z=1 / C$. The exponents at the 
regular singular point $z=1 / C$ of the differential equation $\mathbf{L} y=0$ (with $\mathbf{L}$ being defined in (1.1)) are $0,1, \ldots, \Phi-2$, and

$$
(\Phi-1)-\sum_{j=1}^{k} \sum_{i=1}^{\varphi\left(N_{j}\right)} \frac{r_{i, j}}{N_{j}}
$$

By the elementary identity

$$
\sum_{j=1}^{k} \sum_{i=1}^{\varphi\left(N_{j}\right)} \frac{r_{i, j}}{N_{j}}=\frac{\Phi}{2}
$$

the value (4.10) simplifies to $\frac{1}{2} \Phi-1$. By the theory of hypergeometric differential equations (see [11, Ch. 4, Section 8]), a basis over $\mathbb{C}$ of solutions of $\mathbf{L}$ consists of $\Phi-1$ functions $f_{1}(z), \ldots, f_{\Phi-1}(z)$ holomorphic at $z=1 / C$, together with another solution $f_{\Phi}(z)$ which can be described as follows.

(a) If $\Phi$ is odd, then $f_{\Phi}(z)=(1-C z)^{\Phi / 2-1} u(z)$, where $u(z)$ is holomorphic at $z=1 / C$.

(b) If $\Phi$ is even, then $f_{\Phi}(z)=v(z)+(1-C z)^{\Phi / 2-1} \log (1-C z) u(z)$, where both $u(z)$ and $v(z)$ are holomorphic at $z=1 / C$.

It follows that, in a neighbourhood of $1 / C$ avoiding the cut $[1 / C,+\infty)$,

$$
\begin{aligned}
\mathbf{F}_{\mathbf{N}}(z) & =f(z)+(1-C z)^{\Phi / 2-1} L(z) g(z) \\
\mathbf{G}_{\mathbf{N}}(z) & =\breve{f}(z)+(1-C z)^{\Phi / 2-1} L(z) \breve{g}(z),
\end{aligned}
$$

where $f, \breve{f}, g$ and $\breve{g}$ are holomorphic around $z=1 / C$, and $L(z)=1$ if $\Phi$ is odd or $L(z)=-\log (1-C z)$ if $\Phi$ is even. Indeed, since the function $\mathbf{G}_{\mathbf{N}}(z)+\log (a z) \mathbf{F}_{\mathbf{N}}(z)$ (with $a \neq 0$ ) is a solution of $\mathbf{L} y=0$, it can be written in a form similar to the righthand side of (4.12). Application of (4.12) to $\log (a z) \mathbf{F}_{\mathbf{N}}(z)$ then gives (4.13) because $\log (a z)$ is holomorphic at $z=1 / C$.

Concerning the coefficients of $\mathbf{F}_{\mathbf{N}}(z)$, by Stirling's formula,

$$
\mathbf{B}_{\mathbf{N}}(m)=\left(\prod_{j=1}^{k} \prod_{i=1}^{\varphi\left(N_{j}\right)} \frac{1}{\Gamma\left(r_{i, j} / N_{j}\right)}\right) \times \frac{C^{m}}{m^{\Phi / 2}}(1+o(1)), \quad m \rightarrow \infty,
$$

which implies that $g(1 / C)>0$ by the classical link between singularities of an analytic function $h$ and the asymptotic behaviour of the Taylor coefficients of $h$ when these are positive (see [14, Ch. VI]).

Concerning the coefficients of $\mathbf{G}_{\mathbf{N}}(z)$,

$$
\mathbf{B}_{\mathbf{N}}(m) \mathbf{H}_{\mathbf{N}}(m)=\mathbf{B}_{\mathbf{N}}(m)\left(\log (C)-\frac{\Phi}{2 m}+O\left(\frac{1}{m^{2}}\right)\right) .
$$

(In fact, $\log (C)$ appears in the form $\sum_{j=1}^{k} \sum_{i=1}^{\varphi\left(N_{j}\right)}\left(\psi(1)-\psi\left(r_{i, j} / N\right)\right)$; see (3.6).) Hence, using (4.15), we can make (4.13) more precise:

$$
\mathbf{G}_{\mathbf{N}}(z)=\log (C) \mathbf{F}_{\mathbf{N}}(z)+\widetilde{f}(z)+(1-C z)^{\Phi / 2} L(z) \widetilde{g}(z)
$$


where $\widetilde{f}$ and $\widetilde{g}$ are holomorphic at $z=1 / C$, and $\widetilde{g}(1 / C) \neq 0$. (This is more precise when one transforms $\log (C) \mathbf{F}_{\mathbf{N}}(z)$ using (4.12).)

In order to proceed, we need the following auxiliary result.

Claim. For any vector $\mathbf{N}$ of positive integers, the limit

$$
S:=\lim _{z \rightarrow 1 / C}\left(\mathbf{G}_{\mathbf{N}}(z)-\log (C) \mathbf{F}_{\mathbf{N}}(z)\right)
$$

exists, is finite and is negative. Furthermore, it is equal to $\widetilde{f}(1 / C)$.

Here and later, the limit $z \rightarrow 1 / C$ is understood along real numbers $z<1 / C$.

In order to see (4.17), we observe that, if $|z|<1 / C$, then

$$
\mathbf{G}_{\mathbf{N}}(z)-\log (C) \mathbf{F}_{\mathbf{N}}(z)=\sum_{m=0}^{\infty} \mathbf{B}_{\mathbf{N}}(m)\left(\mathbf{H}_{\mathbf{N}}(m)-\log (C)\right) z^{m} .
$$

The series on the right-hand side converges if $z=1 / C$ because

$$
\left|\mathbf{B}_{\mathbf{N}}(m)\left(\mathbf{H}_{\mathbf{N}}(m)-\log (C)\right)\right|=O\left(\frac{C^{m}}{m^{\Phi / 2+1}}\right)
$$

and $\frac{1}{2} \Phi+1>1$. By Abel's theorem, the limit $S$ in (4.17) exists and

$$
S=\sum_{m=0}^{\infty} \mathbf{B}_{\mathbf{N}}(m)\left(\mathbf{H}_{\mathbf{N}}(m)-\log (C)\right) C^{-m},
$$

the right-hand side being finite.

Second, since $H(x, n)=\psi(n+x)-\psi(x)$, it is easy to see that

$$
\mathbf{H}_{\mathbf{N}}(m)-\log (C)=\sum_{j=1}^{k} \sum_{i=1}^{\varphi\left(N_{j}\right)}\left(\psi\left(m+\frac{r_{i, j}}{N_{j}}\right)-\psi(m+1)\right),
$$

where we have used (3.6) again. Since the function $\psi$ is strictly increasing on $(0,+\infty)$, and since $0<r_{i, j} / N<1$, we deduce that

$$
\mathbf{H}_{\mathbf{N}}(m)-\log (C)<0 \quad \forall m \geq 0 .
$$

Hence $S<0$.

Finally, since $\Phi / 2>0$, equation (4.16) implies that

$$
\lim _{z \rightarrow 1 / C}\left(\mathbf{G}_{\mathbf{N}}(z)-\log (C) \mathbf{F}_{\mathbf{N}}(z)\right)=\lim _{z \rightarrow 1 / C}\left(\widetilde{f}(z)+(1-C z)^{\Phi / 2} L(z) \widetilde{g}(z)\right)=\widetilde{f}(1 / C),
$$

thus completing the proof of (4.17).

We may now continue with the proof of Lemma 4.3. By the remarks preceding (4.17),

$$
\frac{\mathbf{G}_{\mathbf{N}}(z)}{\mathbf{F}_{\mathbf{N}}(z)}=\log (C)+\frac{\widetilde{f}(z)+(1-C z)^{\Phi / 2} L(z) \widetilde{g}(z)}{f(z)+(1-C z)^{\Phi / 2-1} L(z) g(z)} .
$$


This is now translated to $\mathbf{q}_{\mathbf{N}}(z)=z \exp \left(\mathbf{G}_{\mathbf{N}}(z) / \mathbf{F}_{\mathbf{N}}(z)\right)$. Let us for the moment restrict ourselves to the case where $\Phi \geq 3$. It was argued in the paragraph between (4.13) and (4.15) that $g(1 / C)>0$. Furthermore, by the definition of $f(z)$ in (4.12), we have $f(1 / C)=\mathbf{F}_{\mathbf{N}}(1 / C) \neq 0$. Finally, by $(4.17), \widetilde{f}(1 / C)<0$. If we use these observations, together with our assumption that $\Phi \geq 3$, from (4.19) we obtain the singular expansion

$$
\begin{aligned}
\mathbf{q}_{\mathbf{N}}(z) & =z \exp \left(\frac{\mathbf{G}_{\mathbf{N}}(z)}{\mathbf{F}_{\mathbf{N}}(z)}\right) \\
& =C z \exp (\widetilde{f}(z) / f(z))\left(1+\alpha_{1}(1-C z)^{\Phi / 2-1} L(z)+O\left((1-C z)^{\Phi / 2} L(z)\right)\right)
\end{aligned}
$$

as $z \rightarrow 1 / C$. Here,

$$
\alpha_{1}=-\exp \left(\frac{\tilde{f}(1 / C)}{f(1 / C)}\right) \frac{g(1 / C) \tilde{f}(1 / C)}{f^{2}(1 / C)}=\mathbf{q}_{\mathbf{N}}(1 / C) \frac{g(1 / C)\left(\mathbf{F}_{\mathbf{N}}(1 / C) \log (C)-\mathbf{G}_{\mathbf{N}}(1 / C)\right)}{\mathbf{F}_{\mathbf{N}}^{2}(1 / C)},
$$

which is positive because of (4.18) and $g(1 / C)>0$. The singular expansions (4.8) and (4.9) now follow routinely: the claim on the sign of $\mathrm{q}_{d+1 / 2}$ and of $\mathrm{q}_{d+}$, respectively, is a direct consequence of $\alpha_{1}$ being positive, while the claim on the sign of $\mathrm{q}_{1}$ follows from the fact that $\mathbf{q}_{\mathbf{N}}(z)$ is monotone increasing on the interval $[0,1 / C]$ (which is implied by Theorem 1.1).

Finally, if $\Phi=2$, then, leaving the details to the reader, the singular expansion in this case is

$$
\mathbf{q}_{\mathbf{N}}(z)=1+\frac{\alpha_{2}}{\log (1-C z)}+O\left(\frac{1}{\log ^{2}(1-C z)}\right)
$$

as $z \rightarrow 1 / C$. Here, $\alpha_{2}=-\widetilde{f}(1 / C) / g(1 / C)$, which is positive by our earlier observations.

The remaining lemmas in this section are general results that describe the singular expansion of a function $z(q)$ at $q=q_{0}$, given the singular expansion of its compositional inverse $q(z)$ at the corresponding point. They are tailor-made for obtaining singular expansions of $\mathbf{z}_{\mathbf{N}}(q)$ at $q=-\exp (-\pi \cot (\pi / M))$ and at $q=\mathbf{q}_{\mathbf{N}}(1 / C)$ by combining the appropriate lemma with Lemma 4.1 and Lemma 4.3, respectively.

We start with the results which, upon combination with Lemma 4.3, imply singular expansions of $\mathbf{z}_{\mathbf{N}}(q)$ at $q=\mathbf{q}_{\mathbf{N}}(1 / C)$. In the statements, we make use of right and left slit neighbourhoods, notions defined in the paragraph before Proposition 1.6.

Lemma 4.4. Suppose that we are given a complex function $q(z)$ which is analytic in a right slit neighbourhood of $z=z_{0}$ and has a singular expansion that begins

$$
\begin{aligned}
q(z)=q_{0} & +q_{1}\left(z-z_{0}\right)+q_{2}\left(z-z_{0}\right)^{2}+\cdots \\
& +q_{d}\left(z-z_{0}\right)^{d}+q_{d+1 / 2}\left(z_{0}-z\right)^{d+1 / 2}+O\left(\left(z-z_{0}\right)^{d+1}\right),
\end{aligned}
$$

where $d \geq 0, q_{d+1 / 2} \neq 0$ and, if $d>0$, then also $q_{1} \neq 0$. Then there exists a local inverse function $z(q)$ which, in a right slit neighbourhood of $q=q_{0}$, is analytic and has a 
singular expansion that begins

$$
z(q)=z_{0}+z_{2}\left(q-q_{0}\right)^{2}+O\left(\left(q-q_{0}\right)^{4}\right)
$$

if $d=0$, where $z_{2}=-1 / q_{1 / 2}^{2}$, and begins

$$
\begin{aligned}
z(q)=z_{0} & +z_{1}\left(q-q_{0}\right)+z_{2}\left(q-q_{0}\right)^{2}+\cdots \\
& +z_{d}\left(q-q_{0}\right)^{d}+z_{d+1 / 2}\left(q_{0}-q\right)^{d+1 / 2}+O\left(\left(q-q_{0}\right)^{d+1}\right)
\end{aligned}
$$

if $d>0$, where $z_{1}=1 / q_{1}$ and $z_{d+1 / 2}=-q_{1}^{-d-3 / 2} q_{d+1 / 2}$.

Proof. We concentrate on the case where $d>0$. By standard bootstrap arguments (see [12, Sections 2.5-2.7]), one sees that, in a right slit neighbourhood of $q_{0}$,

$$
z(q)=z_{0}+z_{1}\left(q-q_{0}\right)+z_{2}\left(q-q_{0}\right)^{2}+\cdots+z_{d}\left(q-q_{0}\right)^{d}+\tilde{z}(q),
$$

where the coefficients $z_{0}, z_{1}, \ldots, z_{d}$ agree with the corresponding Taylor coefficients of the compositional inverse of the truncated series

$$
q_{0}+q_{1}\left(z-z_{0}\right)+q_{2}\left(z-z_{0}\right)^{2}+\cdots+q_{d}\left(z-z_{0}\right)^{d},
$$

and where $\tilde{z}(q)$ is a function which is analytic in the same slit neighbourhood, and which satisfies $\tilde{z}(q)=o\left(\left(q-q_{0}\right)^{d}\right)$. In particular, $z_{1}=1 / q_{1}$. Continuing the bootstrap, the expansion (4.21) (with $q$ replaced by $x$ in order to minimise the possible confusion) is now substituted in (4.20). In that manner, we obtain

$$
0=q_{1} \tilde{z}(x)+q_{d+1 / 2} z_{1}^{d+1 / 2}\left(q_{0}-x\right)^{d+1 / 2}+O\left(\left(x-q_{0}\right)^{d+1}\right) .
$$

The claimed result is now obvious.

Lemma 4.5. Suppose that we are given a complex function $q(z)$ which is analytic in a right slit neighbourhood of $z=z_{0}$, where it has a singular expansion that begins

$$
\begin{aligned}
q(z)=q_{0} & +q_{1}\left(z-z_{0}\right)+q_{2}\left(z-z_{0}\right)^{2}+\cdots \\
& +q_{d-1}\left(z-z_{0}\right)^{d-1}+q_{d+}\left(z-z_{0}\right)^{d} \log \left(z_{0}-z\right)+O\left(\left(z-z_{0}\right)^{d}\right),
\end{aligned}
$$

where $d \geq 1, q_{d+} \neq 0$, and, if $d>1$, then also $q_{1} \neq 0$. Then there exists a local inverse function $z(q)$ which, in a right slit neighbourhood of $q=q_{0}$, is analytic and has a singular expansion that begins

$$
z(q)=z_{0}+z_{1+}\left(q-q_{0}\right) \log ^{-1}\left(q_{0}-q\right)+o\left(\left(q-q_{0}\right) \log ^{-1}\left(q_{0}-q\right)\right)
$$

if $d=1$, where $z_{1+}=1 / q_{1+}$, and begins

$$
\begin{aligned}
z(q)=z_{0} & +z_{1}\left(q-q_{0}\right)+z_{2}\left(q-q_{0}\right)^{2}+\cdots \\
& +z_{d-1}\left(q-q_{0}\right)^{d-1}+z_{d+}\left(q-q_{0}\right)^{d} \log \left(q_{0}-q\right)+O\left(\left(q-q_{0}\right)^{d}\right)
\end{aligned}
$$

if $d>1$, where $z_{1}=1 / q_{1}$ and $z_{d+}=-q_{1}^{-d-1} q_{d+}$. 
Proof. For (4.24), we proceed exactly in the same fashion as in the proof of Lemma 4.4.

In order to establish the expansion (4.23), we replace $z$ by $z(x)$ in (4.22), and then take the logarithm on both sides. This leads to

$$
\log \left(q_{0}-x\right)=\log \left(q_{1+}\right)+\log \left(z_{0}-z(x)\right)+\log \log \left(z_{0}-z(x)\right)+O\left(\log ^{-1}\left(z_{0}-z(x)\right)\right) .
$$

In order to simplify, we replace $q_{0}-x$ by $X$ and $\log \left(z_{0}-z(x)\right)$ by $Z(X)$ :

$$
\log (X)=\log \left(q_{1+}\right)+Z(X)+\log (Z(X))+O\left(Z(X)^{-1}\right) .
$$

Applying bootstrapping again in a neighbourhood of $X=0$, we must have $Z(X)=$ $\log (X)+\tilde{Z}(X)$, where $\tilde{Z}(X)=o(\log (X))$. If we substitute this in (4.25), we obtain, after a little simplification,

$$
0=\log \left(q_{1+}\right)+\tilde{Z}(X)+\log \log (X)+o(1) .
$$

Now we see that $\tilde{Z}(X)=-\log \log (X)+\tilde{\tilde{Z}}(X)$, where $\tilde{\tilde{Z}}(X)=o(\log \log (X))$. By substituting this in (4.26), we arrive at

$$
0=\log \left(q_{1+}\right)+\tilde{\tilde{Z}}(X)+o(1)
$$

from which we deduce that $\tilde{Z}(X)=-\log \left(q_{1+}\right)+o(1)$. If we now put everything together, then we obtain

$$
Z(X)=\log (X)-\log \log (X)-\log \left(q_{1+}\right)+o(1),
$$

or, in the original notation,

$$
z_{0}-z(x)=\frac{q_{0}-x}{q_{1+} \log \left(q_{0}-x\right)}(1+o(1))
$$

After replacement of $x$ by $q$, we see that this is equivalent to (4.23).

LEMMA 4.6. Suppose that we are given a complex function $q(z)$ which is analytic in a right slit neighbourhood of $z=z_{0}$, where it has a singular expansion that begins

$$
q(z)=q_{0}+q_{1} \log ^{-1}\left(z_{0}-z\right)+O\left(\log ^{-2}\left(z_{0}-z\right)\right),
$$

where $q_{1} \neq 0$. Then there exists a local inverse function $z(q)$ which, in a right slit neighbourhood of $q=q_{0}$, is analytic and has a singular expansion of the form

$$
z(q)=\exp \left(\frac{q_{1}}{q-q_{0}}+O(1)\right) .
$$

In particular, $z(q)$ has an essential singularity at $q=q_{0}$.

Proof. This is again easily derived by bootstrapping. 
Finally we provide a general result which, upon combination with Lemma 4.1, yields the singular expansion of $\mathbf{z}_{\mathbf{N}}(q)$ at $q=-\exp (-\pi \cot (\pi / M))$.

Lemma 4.7. Let $q(z)$ be a complex function that is analytic near $\infty$ except for a cut on the positive real axis.

(i) Suppose that $q(z)$ has a singular expansion at $z=\infty$ that begins

$$
q(z)=q_{0}+q_{1}(-z)^{-r / n}+O\left((-z)^{-(r+1) / n} \log ^{b}(-z)\right),
$$

where $q_{1} \neq 0$ and $r, n, b$ are nonnegative integers such that $r \geq 1$. Then there exists a local inverse function $z(q)$ which, in a neighbourhood of $q=q_{0}$, has a singular expansion that begins

$$
z(q)=-q_{1}^{n / r}\left(q-q_{0}\right)^{-n / r}+O\left(\left(q-q_{0}\right)^{-(n-1) / r} \log ^{b}\left(q_{0}-q\right)\right) .
$$

(ii) Suppose that $q(z)$ has a singular expansion at $z=\infty$ that begins

$$
q(z)=q_{0}+q_{1}(-z)^{-r / n} \log ^{b}(-z)+O\left((-z)^{-r / n} \log ^{b-1}(-z)\right),
$$

where $q_{1} \neq 0$ and $r, n, b$ are nonnegative integers with $r, b \geq 1$. Then there exists a local inverse function $z(q)$ which, in a neighbourhood of $q=q_{0}$, has a singular expansion that begins

$$
\begin{gathered}
z(q)=-\left(-\frac{n}{r}\right)^{b r / n} q_{1}^{n / r}\left(q-q_{0}\right)^{-n / r} \log ^{b n / r}\left(q_{0}-q\right) \\
+O\left(\left(q-q_{0}\right)^{-n / r} \log ^{b n / r-1}\left(q_{0}-q\right)\right) .
\end{gathered}
$$

(iii) Suppose that $q(z)$ has a singular expansion at $z=\infty$ that begins

$$
q(z)=q_{0}+q_{1} \log ^{-1}(-z)+O\left(\log ^{-2}(-z)\right),
$$

where $q_{1} \neq 0$. Then there exists a local inverse function $z(q)$ which, in a neighbourhood of $q=q_{0}$, has a singular expansion that begins

$$
z(q)=\exp \left(\frac{q_{1}}{q-q_{0}}+O(1)\right)
$$

REMARK 4.8. If, in case (i) with $r=1$, the series $q(z)$ in fact admits a Puiseux expansion in $(-z)^{-1 / n}$, then it is not difficult to see that $z(q)$ has a pole of order $n$ at $q=q_{0}$. If the singular expansion of $q(z)$ at $z=-\infty$ has terms containing $\log (-z)$ (which is often the case in the situation of Lemma 4.1; see the remark accompanying that lemma), then the point $q=q_{0}$ will be a branch point of $z(q)$.

Proof. Once more, this is easily derived by bootstrapping. 


\section{Proof of Theorem 1.2}

For the proof of the theorem, we shall require the following auxiliary result.

Lemma 5.1. The function $\mathbf{F}_{\mathbf{N}}(z)$ does not vanish on its disk of convergence $|z|<1 / C_{\mathbf{N}}$.

Proof. The following argument is borrowed from [22, p. 94, last corollary]. By Corollary 2.4, the Taylor coefficients $\widehat{\mathbf{B}}_{\mathbf{N}}(m)$ of $\widehat{\mathbf{F}}_{\mathbf{N}}(z)$ are positive and satisfy equation (2.1), that is,

$$
\delta_{n, 0}+\sum_{k=1}^{n} \widehat{\mathbf{B}}_{\mathbf{N}}(k) \mathbf{B}_{\mathbf{N}}(n-k)=\mathbf{B}_{\mathbf{N}}(n) .
$$

Since the coefficients $\mathbf{B}_{\mathbf{N}}(m)$ are also positive and $\mathbf{B}_{\mathbf{N}}(0)=1$, it follows that $0 \leq$ $\widehat{\mathbf{B}}_{\mathbf{N}}(n) \leq \mathbf{B}_{\mathbf{N}}(n)$ whenever $n \geq 0$. Hence, the radius of convergence of the Taylor series of $1 / \mathbf{F}_{\mathbf{N}}(z)$ at $z=0$ is at least as large as the radius of convergence of $\mathbf{F}_{\mathbf{N}}(z)$ at $z=0$. It is necessarily equal to the latter because $z=1 / C$ is a branch point of $\mathbf{F}_{\mathbf{N}}(z)$, and thus also of $1 / \mathbf{F}_{\mathbf{N}}(z)$.

In particular, $\mathbf{F}_{\mathbf{N}}(z)$ cannot vanish at some point $z_{0}$ such that $\left|z_{0}\right|<1 / C$, because otherwise the radius of convergence of $1 / \mathbf{F}_{\mathbf{N}}(z)$ would be at most $\left|z_{0}\right|$, which gives a contradiction.

Remark 5.2. In the proof of Theorem 1.3(i) (given in Section 6), we show that a classical result of Pólya [29] on hypergeometric series implies that $\mathbf{F}_{\mathbf{N}}(z)$ vanishes nowhere in the slit plane $\mathbb{C} \backslash[1 / C,+\infty)$. Nevertheless, we believe that the above argument, proving a weaker result, is still worth recording since it is based only on the positivity of the coefficients $\mathbf{B}_{\mathbf{N}}(m)$ and $\widehat{\mathbf{B}}_{\mathbf{N}}(m)$ and not on the hypergeometric nature of $\mathbf{F}_{\mathbf{N}}(z)$.

We now turn to the proof of Theorem 1.2.

(i), (ii) In view of the explicit expression (1.4), the claim is trivial for $\Phi=1$. We therefore assume $\Phi \geq 2$ from now on.

Clearly, the discussion in the proof of Lemma 5.1 also implies that the radius of convergence of the Taylor series at $z=0$ of $\exp \left(\mathbf{G}_{\mathbf{N}}(z) / \mathbf{F}_{\mathbf{N}}(z)\right)$ is at least $1 / C$. By Lemma 4.3 , which says in particular that $\mathbf{q}_{\mathbf{N}}(z)$ has a singularity at $z=1 / C$, it cannot be larger.

It remains to prove that the Taylor series $\sum_{m \geq 1} \mathbf{q}_{m} z^{m}$ of $\mathbf{q}_{\mathbf{N}}(z)$ converges on the circle $|z|=1 / C$. By (4.17),

$$
\begin{aligned}
\lim _{z \rightarrow 1 / C} \frac{\mathbf{G}_{\mathbf{N}}(z)}{\mathbf{F}_{\mathbf{N}}(z)} & =\log (C)+\lim _{z \rightarrow 1 / C} \frac{\mathbf{G}_{\mathbf{N}}(z)-\log (C) \mathbf{F}_{\mathbf{N}}(z)}{\mathbf{F}_{\mathbf{N}}(z)} \\
& =\log (C)+\frac{S}{\mathbf{F}_{\mathbf{N}}(1 / C)},
\end{aligned}
$$

where the second term must be understood as 0 if $\lim _{z \rightarrow 1 / C} \mathbf{F}_{\mathbf{N}}(z)=+\infty$. Hence $\mathbf{q}_{\mathbf{N}}(1 / C)$ exists and is finite. By Abel's theorem, $\mathbf{q}_{\mathbf{N}}(1 / C)=\sum_{m \geq 1} \mathbf{q}_{m} / C^{m}$, and since the $\mathrm{q}_{m}$ are nonnegative, $\sum_{m \geq 1} \mathrm{q}_{m} z^{m}$ converges for any $z$ such that $|z|=1 / C$. 
(iii) We have

$$
\max _{|z|=1 / C}\left|\mathbf{q}_{\mathbf{N}}(z)\right|=\mathbf{q}_{\mathbf{N}}(1 / C) .
$$

By (5.1),

$$
\mathbf{q}_{\mathbf{N}}(1 / C)=\exp \left(\frac{S}{\mathbf{F}_{\mathbf{N}}(1 / C)}\right) \leq 1
$$

because $S<0$ and $\mathbf{F}_{\mathbf{N}}(1 / C)>0$. Equality holds only if $\mathbf{F}_{\mathbf{N}}(1 / C)=+\infty$, which, by (4.14), happens exactly when $\Phi=1$ or $\Phi=2$.

(iv) The claimed assertions follow from (1.4) after a little calculation.

(v) By the standard theorems of singularity analysis (see [14, Ch. VI]), the assertion follows immediately from Lemma 4.3(i).

(vi) Again, by the standard theorems of singularity analysis, the assertion follows immediately from Lemma 4.3(ii), (iii).

\section{Proof of Theorem 1.3}

(i) From Section 3, we know that $\mathbf{F}_{\mathbf{N}}(z)$ and $\mathbf{G}_{\mathbf{N}}(z)$ can both be analytically continued to $\mathbb{C} \backslash[1 / C,+\infty)$. Hence

$$
\mathbf{q}_{\mathbf{N}}(z)=\exp \left(\frac{\mathbf{G}_{\mathbf{N}}(z)+\log (z) \mathbf{F}_{\mathbf{N}}(z)}{\mathbf{F}_{\mathbf{N}}(z)}\right)=z \exp \left(\frac{\mathbf{G}_{\mathbf{N}}(z)}{\mathbf{F}_{\mathbf{N}}(z)}\right)
$$

can be continued at least to $\mathbb{C} \backslash([1 / C,+\infty) \cup Z)$, where $Z$ is the set of zeros of $\mathbf{F}_{\mathbf{N}}(z)$.

The reader should recall that Lemma 5.1 says that the intersection of $Z$ and the open disk of convergence of $\mathbf{F}_{\mathbf{N}}(z)$ is empty. We now show that, in fact, the entire set $Z$ is empty. For this, we apply a result of Pólya [29, p. 192] on hypergeometric functions. Recall the hypergeometric notation

$$
{ }_{q+1} F_{q}\left[\begin{array}{c}
a_{0}, a_{1}, \ldots, a_{q} \\
b_{1}, \ldots, b_{q}
\end{array} z\right]=\sum_{k=0}^{\infty} \frac{\left(a_{0}\right)_{k}\left(a_{1}\right)_{k} \cdots\left(a_{q}\right)_{k}}{k !\left(b_{1}\right)_{k} \cdots\left(b_{q}\right)_{k}} z^{k} .
$$

Pólya's result implies in particular that the above hypergeometric function does not vanish for any $z \in \mathbb{C} \backslash[1,+\infty)$ when $0<a_{0}<1,0<a_{1}<b_{1}, \ldots, 0<a_{q}<b_{q}$. Now, indeed, $\mathbf{F}_{\mathbf{N}}(z)$ can be written in hypergeometric notation:

$$
\mathbf{F}_{\mathbf{N}}(z)={ }_{\Phi} F_{\Phi-1}\left[\begin{array}{c}
r_{1,1} / N_{1}, \ldots, r_{k, \varphi\left(N_{k}\right)} / N_{k} \\
1, \ldots, 1
\end{array} C z .\right.
$$

In particular, we see that Pólya's conditions are satisfied by this hypergeometric function, which proves that $Z$ is empty.

(ii) If $\Phi \geq 2$, this is a consequence of the singular expansion of $\mathbf{q}_{\mathbf{N}}(z)$ at $z=1 / C$ given in Lemma 4.3.

If $\Phi=1$, then we know that $\mathbf{q}_{(2)}(z)=(1-\sqrt{1-4 z})^{2} /(4 z)$, which evidently has a branch point with nontrivial monodromy at $z=1 / C_{(2)}=1 / 4$. 
(iii) The assertion concerning the limit of $\mathbf{q}_{\mathbf{N}}(z)$ at infinity is an immediate consequence of Lemma 3.1.

\section{Proof of Theorem 1.4}

(i) We know from Theorem 1.3 that

$$
\lim _{z \rightarrow \infty} \mathbf{q}_{\mathbf{N}}(z)=-\exp (-\pi \cot (\pi / M))=: \rho
$$

where the limit has to be taken along a path that avoids the cut $[1 / C,+\infty)$. Let us suppose that the radius of convergence of $\mathbf{z}_{\mathbf{N}}(q), R$ say, is strictly larger than $|\rho|$. One can find $\varepsilon>0$ and $A(\varepsilon)>0$ with the property that, if $|x|>A(\varepsilon)$ and $x \notin[1 / C,+\infty)$, then

$$
\left|\mathbf{q}_{\mathbf{N}}(x)-\rho\right|<\varepsilon \quad \text { and } \quad|\rho|+\varepsilon<R .
$$

For the above $x$, the quantity $\mathbf{z}_{\mathbf{N}}\left(\mathbf{q}_{\mathbf{N}}(x)\right)$ is well defined and is equal to $x$. Consequently,

$$
\infty=\lim _{\substack{x \rightarrow \infty \\|x|>A(\varepsilon) \\ x \notin[1 / C,+\infty)}} x=\lim _{\substack{x \rightarrow \infty \\|x|>A(\varepsilon) \\ x \notin[1 / C,+\infty)}} \mathbf{z}_{\mathbf{N}}\left(\mathbf{q}_{\mathbf{N}}(x)\right)=\lim _{q \rightarrow \rho} \mathbf{z}_{\mathbf{N}}(q),
$$

where the last limit is along a suitable path. Hence, the point $q=\rho$ is a singularity of $\mathbf{z}_{\mathbf{N}}(q)$, which contradicts our assumption that $R>|\rho|$. Therefore the radius of convergence $R$ is in fact no more than $|\rho|$.

(ii) Arguing by contradiction, we suppose that the radius of convergence of $\mathbf{z}_{\mathbf{N}}(q)$ is strictly larger than $\mathbf{q}_{\mathbf{N}}(1 / C)$. In that case, $\mathbf{z}_{\mathbf{N}}(q)$ is analytic around $\mathbf{q}_{\mathbf{N}}(1 / C)$. For the derivative of $\mathbf{z}_{\mathbf{N}}$, we have

$$
\mathbf{z}_{\mathbf{N}}^{\prime}\left(\mathbf{q}_{\mathbf{N}}(z)\right)=\frac{1}{\mathbf{q}_{\mathbf{N}}^{\prime}(z)}
$$

Let us first assume that $\Phi>4$. From Theorem 1.2(vi), we know that the $m$ th coefficient of $\mathbf{q}_{\mathbf{N}}(z), \mathbf{q}_{m}$ say, behaves like $C^{m} / m^{\Phi / 2}$ (up to a multiplicative constant). Hence, since $\Phi / 2>2$, the series $\sum_{m=0}^{\infty} m \mathrm{q}_{m} / C^{m-1}$ converges, and by Abel's theorem it agrees with the limit

$$
\omega_{1}:=\lim _{\substack{z \rightarrow 1 / C \\|z|<1 / C}} \mathbf{q}_{\mathbf{N}}^{\prime}(z)
$$

Hence, by (7.1) and the continuity of $\mathbf{z}_{\mathbf{N}}^{\prime}(q)$, we have $\mathbf{z}_{\mathbf{N}}^{\prime}\left(\mathbf{q}_{\mathbf{N}}(1 / C)\right)=1 / \omega_{1}$, which is different from zero. As a consequence, by [17, Theorems 2.4b, 2.4c], $\mathbf{z}_{\mathbf{N}}(q)$ can be inverted in a neighbourhood of $q=\mathbf{q}_{\mathbf{N}}(1 / C)$. This would imply that $\mathbf{q}_{\mathbf{N}}(z)$ is analytic around $1 / C$, which contradicts Theorem 1.2(ii).

On the other hand, if $\Phi=4$, the above argument has to be adapted in order to lead to a contradiction. To begin with, by Lemma 4.3(iii),

$$
\mathbf{q}_{\mathbf{N}}(z)-\mathbf{q}_{\mathbf{N}}(1 / C)=\omega_{2}(1-C z) \log (1-C z)(1+o(1))
$$


as $z \rightarrow 1 / C$, where $\omega_{2}$ is some nonzero constant. Next, we compute the derivative of $\mathbf{q}_{\mathbf{N}}(z)$ using the expression given in (4.19) for the quotient $\mathbf{G}_{\mathbf{N}}(z) / \mathbf{F}_{\mathbf{N}}(z)$. Subsequently, we compute its singular expansion as $z \rightarrow 1 / C$ in the same style as that for $\mathbf{q}_{\mathbf{N}}(z)$. The result is that

$$
\mathbf{q}_{\mathbf{N}}^{\prime}(z)=\omega_{3} \log (1-C z)(1+o(1))
$$

as $z \rightarrow 1 / C$, where $\omega_{3}$ is some nonzero constant. Consequently,

$$
\mathbf{z}_{\mathbf{N}}^{\prime}\left(\mathbf{q}_{\mathbf{N}}(1 / C)\right)=\lim _{\substack{z \rightarrow 1 / C \\|z|<1 / C}} \frac{1}{\mathbf{q}_{\mathbf{N}}^{\prime}(z)}=0 .
$$

Since $\mathbf{z}_{\mathbf{N}}^{\prime}(q)$ is analytic in a neighbourhood of $q=\mathbf{q}_{\mathbf{N}}(1 / C)$, and since $\mathbf{z}_{\mathbf{N}}^{\prime}(q)$ cannot be constant (this would imply that $\mathbf{q}_{\mathbf{N}}(z)$ is linear, which would contradict Theorem 1.2(ii)),

$$
\mathbf{z}_{\mathbf{N}}^{\prime}(q)=\omega_{4}\left(q-\mathbf{q}_{\mathbf{N}}(1 / C)\right)^{s}(1+o(1))
$$

as $q \rightarrow \mathbf{q}_{\mathbf{N}}(1 / C)$, where $\omega_{4}$ is a nonzero constant and $s$ is a positive integer. If we use this in (7.1), together with (7.2) and (7.3), we obtain

$$
\omega_{4} \omega_{2}^{s}(1-C z)^{s} \log ^{s}(1-C z)(1+o(1))=\frac{1+o(1)}{\omega_{3} \log (1-C z)}
$$

as $z \rightarrow 1 / C$, which is absurd.

Remark 7.1. The above argument for the case where $\Phi=4$ does not lead to a contradiction when applied to the case where $\Phi=3$. This is in accordance with Theorem 1.5 and the fact that $\exp (-\pi \cot (\pi / M))$ is larger than $\mathbf{q}_{\mathbf{N}}\left(1 / C_{\mathbf{N}}\right)$ in the relevant cases; see Section 9.

\section{Comparison of the two critical values $\exp \left(-\pi \cot \left(\pi / M_{\mathrm{N}}\right)\right)$ and $\mathrm{q}_{\mathrm{N}}\left(1 / C_{\mathrm{N}}\right)$}

Because of (1.6), the point $q=\exp \left(-\pi \cot \left(\pi / M_{\mathrm{N}}\right)\right)$ is a potential singularity of $\mathbf{z}_{\mathbf{N}}(q)$. In Theorem 1.4(ii) we have shown that the radius of convergence of $\mathbf{z}_{\mathbf{N}}(q)$ is at most $\mathbf{q}_{\mathbf{N}}\left(1 / C_{\mathbf{N}}\right)$. It is therefore important to know whether $\exp \left(-\pi \cot \left(\pi / M_{\mathbf{N}}\right)\right)$ is less than $\mathbf{q}_{\mathbf{N}}\left(1 / C_{\mathbf{N}}\right)$ or not. In this section, we show that when $\Phi_{\mathbf{N}} \geq 4$, we in fact get $\exp \left(-\pi \cot \left(\pi / M_{\mathbf{N}}\right)\right)>\mathbf{q}_{\mathbf{N}}\left(1 / C_{\mathbf{N}}\right)$, which fits well with Conjecture 1.8.

Lemma 8.1. Let $N_{1}, N_{2}, \ldots, N_{k}$ be positive integers, all of which are at least 2 , such that $\Phi=\Phi_{\mathbf{N}} \geq 4$. Furthermore, let $M=M_{\mathbf{N}}$, as before. Then

$$
\exp (-\pi \cot (\pi / M))>\mathbf{q}_{\mathbf{N}}(1 / C) .
$$

Proof. From the inequality at the top of page 157 of [32], which, as the proof shows, remains valid for real $n$, we know that, when $x \geq 1$,

$$
\Gamma(x+1)=\sqrt{2 \pi x}\left(\frac{x}{e}\right)^{x} e^{\lambda_{x}} \quad \text { where } \frac{1}{12 x+1}<\lambda_{x}<\frac{1}{12 x} .
$$


We use these effective bounds on the gamma function to provide an upper bound for $\mathbf{B}_{\mathbf{N}}(m)$. Let us first suppose that $m \geq 2$. Then, putting $s_{i, j}=r_{i, j} / N_{j}$,

$$
\begin{aligned}
\mathbf{B}_{\mathbf{N}}(m) & =\prod_{j=1}^{k} \mathbf{B}_{N_{j}}(m)=C^{m} \prod_{j=1}^{k} \prod_{i=1}^{\varphi\left(N_{j}\right)} \frac{\left(s_{i, j}\right)_{m}}{m !} \\
& =C^{m} \prod_{j=1}^{k} \prod_{i=1}^{\varphi\left(N_{j}\right)} \frac{\Gamma\left(m+s_{i, j}\right)}{\Gamma\left(s_{i, j}\right) \Gamma(m+1)} \\
& <C^{m} e^{\Lambda_{m}} \prod_{j=1}^{k} \prod_{i=1}^{\varphi\left(N_{j}\right)} \frac{1}{\Gamma\left(s_{i, j}\right)}\left(\frac{m+s_{i, j}-1}{m}\right)^{m+1 / 2}\left(\frac{m+s_{i, j}-1}{e}\right)^{s_{i, j}-1},
\end{aligned}
$$

where

$$
\Lambda_{m}=\sum_{j=1}^{k} \sum_{i=1}^{\varphi\left(N_{j}\right)}\left(\frac{1}{12\left(m+s_{i, j}-1\right)}-\frac{1}{12 m+1}\right) .
$$

The quantity $\Lambda_{m}$ may be estimated from above as follows:

$$
\begin{aligned}
\Lambda_{m} & =\sum_{j=1}^{k} \sum_{i=1}^{\varphi\left(N_{j}\right)}\left(\frac{13-12 s_{i, j}}{12\left(m+s_{i, j}-1\right)(12 m+1)}\right) \leq \sum_{j=1}^{k} \sum_{i=1}^{\varphi\left(N_{j}\right)}\left(\frac{13-12 s_{i, j}}{12(m-1)(12 m+1)}\right) \\
& \leq \sum_{j=1}^{k} \varphi\left(N_{j}\right)\left(\frac{13-6}{12(m-1)(12 m+1)}\right) \leq \frac{7 \Phi}{12(m-1)(12 m+1)} \leq \frac{7}{300} \Phi .
\end{aligned}
$$

On the other hand, using the well-known elementary inequality $(1+x / m)^{m} \leq e^{x}$, valid when $x>-m$,

$$
\begin{aligned}
\left(\frac{m+s_{i, j}-1}{m}\right)^{m+1 / 2}\left(\frac{m+s_{i, j}-1}{e}\right)^{s_{i, j}-1} & =\left(1+\frac{s_{i, j}-1}{m}\right)^{m} \frac{e^{1-s_{i, j}}\left(1+\left(s_{i, j}-1\right) / m\right)^{1 / 2}}{\left(m+s_{i, j}-1\right)^{1-s_{i, j}}} \\
& \leq \frac{1}{(m-1)^{1-s_{i, j}}}
\end{aligned}
$$

Finally, recall the reflection formula for the gamma function (see [3, Theorem 1.2.1]), $\Gamma(x) \Gamma(1-x)=\pi / \sin (\pi x)$. If all the $N_{j}$ are different from 2 (in which case $\varphi\left(N_{j}\right)$ is always even), the reflection formula shows that

$$
\prod_{j=1}^{k} \prod_{i=1}^{\varphi\left(N_{j}\right)} \frac{1}{\Gamma\left(s_{i, j}\right)}=\prod_{j=1}^{k} \prod_{i=1}^{\varphi\left(N_{j}\right) / 2} \frac{\sin \left(\pi s_{i, j}\right)}{\pi} \leq \pi^{-\Phi / 2} .
$$

One can then see that the inequality above holds even if some of the $N_{j}$ are equal to 2 . If (8.3), (8.4) and (8.5) are substituted back in (8.2), and if in addition (4.11) is used to compute the arising sum $\sum_{j=1}^{k} \sum_{i=1}^{\varphi\left(N_{j}\right)}\left(1-s_{i, j}\right)$, then the result is

$$
\mathbf{B}_{\mathbf{N}}(m)<C^{m} e^{7 \Phi / 300} \pi^{-\Phi / 2}(m-1)^{-\Phi / 2} .
$$


The case of $m=1$ has to be treated separately. In that case,

$$
\mathbf{B}_{\mathbf{N}}(1)=\prod_{j=1}^{k} \mathbf{B}_{N_{j}}(1)=C \prod_{j=1}^{k} \prod_{i=1}^{\varphi\left(N_{j}\right)} \frac{r_{i, j}}{N_{j}}=C \prod_{j=1}^{k} \prod_{i=1}^{\varphi\left(N_{j}\right) / 2} \frac{r_{i, j}\left(N-r_{i, j}\right)}{N_{j}^{2}} \leq C \times 2^{-\Phi}
$$

if all the $N_{j}$ are different from 2 . Again, it is readily seen that the inequality also holds if some of the $N_{j}$ are equal to 2 .

We now combine (8.6) and (8.7) in order to estimate $\mathbf{F}_{\mathbf{N}}(1 / C)$ :

$$
\begin{aligned}
\mathbf{F}_{\mathbf{N}}(1 / C) & =\sum_{m=0}^{\infty} \mathbf{B}_{\mathbf{N}}(m) C^{-m} \\
& <1+\frac{1}{2^{\Phi}}+\left(\frac{e^{7 / 300}}{\pi^{1 / 2}}\right)^{\Phi} \sum_{m=2}^{\infty}(m-1)^{-\Phi / 2} \\
& <1+\frac{1}{2^{\Phi}}+\left(\frac{e^{7 / 300}}{\pi^{1 / 2}}\right)^{\Phi} \zeta(\Phi / 2) \\
& <1+\frac{1}{2^{\max \{4, k\}}}+\left(\frac{e^{7 / 300}}{\pi^{1 / 2}}\right)^{\max \{4, k\}} \zeta(\max \{4, k\} / 2) .
\end{aligned}
$$

Henceforth, we denote the quantity on the right-hand side of (8.8) by $c_{k}$.

We are now in the position to establish inequality (8.1) for 'sufficiently large' $\mathbf{N}$. Namely, since

$$
\pi \cot (\pi / M) \leq M
$$

and (see (4.18))

$$
\mathbf{G}_{\mathbf{N}}(1 / C)-\log (C) \mathbf{F}_{\mathbf{N}}(1 / C)<-\log (C),
$$

it suffices to prove the inequality

or, equivalently,

$$
\exp (-M) \geq \exp \left(-\frac{\log C}{\mathbf{F}_{\mathbf{N}}(1 / C)}\right)
$$

$$
\log C \geq M \times \mathbf{F}_{\mathbf{N}}(1 / C)
$$

We now make use of the estimate (see [5, Theorem 8.8.7])

$$
\varphi(n) \geq \max \left\{1, \frac{n}{e^{\gamma} \log \log n+3 / \log \log n}\right\}
$$

for the totient function, where $\gamma$ denotes Euler's constant. For convenience, let us write $\bar{\varphi}(n)$ for the right-hand side of (8.9). Then, use of (8.9) in the definition of $C$ gives

$$
\log C \geq \sum_{j=1}^{k} \log N_{j}^{\varphi\left(N_{j}\right)}=\sum_{j=1}^{k} \varphi\left(N_{j}\right) \log N_{j} \geq \sum_{j=1}^{k} \bar{\varphi}\left(N_{j}\right) \log N_{j} .
$$

If we put this together with (8.8), we see that (8.1) will be proved whenever

$$
\sum_{j=1}^{k} \bar{\varphi}\left(N_{j}\right) \log N_{j} \geq c_{k} M
$$


It remains to consider the cases where (8.10) does not hold. We claim that this is only a finite number of cases. Indeed, for fixed $k$, there can only be a finite number of $k$ tuples $\left(N_{1}, N_{2}, \ldots, N_{k}\right)$ for which (8.10) is violated, since trivially $c_{k}$ is constant for fixed $k$, and since $\bar{\varphi}(M) \log M$ grows faster than $M$. On the other hand, if $k \geq 15$, then (8.10) holds automatically. For, we have

$$
\min _{M \geq 2}\left\{\bar{\varphi}(M) \log M-c_{15} M\right\} \geq-9,
$$

and therefore (assuming that $M=N_{1}$ without loss of generality), for $k \geq 15$,

$$
\begin{aligned}
\sum_{j=1}^{k} \bar{\varphi}\left(N_{j}\right) \log N_{j} & =\bar{\varphi}(M) \log M+\sum_{j=2}^{k} \bar{\varphi}\left(N_{j}\right) \log N_{j} \\
& \geq c_{15} M-9+(k-1) \log 2 \\
& \geq c_{k} M-9+(k-1) \log 2 \\
& \geq c_{k} M
\end{aligned}
$$

where we have used the simple fact that the $c_{k}$ are monotone decreasing in $k$ in the third line.

In summary, there is indeed only a finite number of cases left where (8.10) does not hold. For these cases, we have verified by computer that inequality (8.1) is satisfied. To carry out this verification, we used the estimation

$$
\begin{aligned}
\mathbf{q}_{\mathbf{N}}(1 / C) & =\exp \left(\frac{\sum_{m=0}^{\infty} \mathbf{B}_{\mathbf{N}}(m)\left(\mathbf{H}_{N}(m)-\log C\right) C^{-m}}{\sum_{m=0}^{\infty} \mathbf{B}_{\mathbf{N}}(m) C^{-m}}\right) \\
& <\exp \left(\frac{\sum_{m=0}^{20} \mathbf{B}_{\mathbf{N}}(m)\left(\mathbf{H}_{N}(m)-\log C\right) C^{-m}}{\sum_{m=0}^{20} \mathbf{B}_{\mathbf{N}}(m) C^{-m}+e^{7 \Phi / 300} \pi^{-\Phi / 2} \sum_{m=21}^{\infty}(m-1)^{-\Phi / 2}}\right) \\
& <\exp \left(\frac{\sum_{m=0}^{20} \mathbf{B}_{\mathbf{N}}(m)\left(\mathbf{H}_{N}(m)-\log C\right) C^{-m}}{\sum_{m=0}^{20} \mathbf{B}_{\mathbf{N}}(m) C^{-m}+e^{7 \Phi / 300} \pi^{-\Phi / 2}(\Phi / 2-1)^{-1} 19^{1-\Phi / 2}}\right)
\end{aligned}
$$

(with the second line following again from (4.18) and (8.6), and the last line from $\left.\sum_{m=21}^{\infty}(m-1)^{-\Phi / 2}<\int_{19}^{\infty} x^{-\Phi / 2} d x\right)$, and we actually compared the left-hand side of (8.1) with the upper bound on the right-hand side given in (8.11). This completes the proof of the lemma.

\section{Proof of Theorem 1.5: the cases where $\Phi_{N}=1,2,3$}

The case where $\Phi=1$ is trivial. We provide the details nevertheless for the sake of completeness. For the cases where $\Phi=2,3$, it is well documented in the literature that the corresponding functions $\mathbf{z}_{\mathbf{N}}(q)$ live in the world of modular forms. We refer 
the reader to $[35,40]$ for reviews of the corresponding classical theory. Despite this, it seems that the questions that we treat in the present paper, that is, questions concerning the analytic nature of the function $\mathbf{z}_{\mathbf{N}}(q)$, have not been recorded in sufficient detail to extract complete information about, for example, the singularities of the function and, thus, of the radius of convergence of the Taylor series at $q=0$. The purpose of this section is to compile the relevant facts, to provide a coherent overview, and to fill possible gaps whenever necessary.

Since we shall make use of them below, we record here some basic facts on modular forms. Given a subgroup $\Gamma$ of the modular group $\mathrm{SL}_{2}(\mathbb{Z})$ and a nonnegative integer $k$, a function $f(\tau)$ from the complex upper half plane $\mathbb{H}$ to the complex numbers is called a modular form of weight $k$ for $\Gamma$ if it is meromorphic on $\mathbb{H}$ and

$$
f(T(\tau))=(c \tau+d)^{k} f(\tau)
$$

for all elements $T=\left(\begin{array}{ll}a & b \\ c & d\end{array}\right)$ of $\Gamma$, where the action of $T$ is defined by $T(\tau)=(a \tau+b) /$ $(c \tau+d)$. Modular forms of weight $k$ for $\Gamma$ satisfy the valence formula (see [31, Theorem 4.1.4])

$$
\sum_{\zeta \in \mathbb{F}} \frac{\operatorname{ord}(f, \zeta)}{\left|\operatorname{stab}_{\zeta}(\widehat{\Gamma})\right|}=\frac{\left|\mathrm{SL}_{2}(\mathbb{Z}) / \widehat{\Gamma}\right|}{12} k
$$

Here:

- $\quad \mathbb{F}$ is a fundamental region for the action of $\Gamma$ on $\mathbb{H}$ to which one adds the cusps (a system of representatives of the orbit of $\infty$ under the action of $\mathrm{SL}_{2}(\mathbb{Z})$ when restricted to $\Gamma$ );

- $\quad \widehat{\Gamma}$ is the mapping group corresponding to $\Gamma$, that is, the group arising from $\Gamma$ upon identification of $T$ and $-T$ if both of them are contained in $\Gamma$ (the action (9.1) on $\mathbb{H}$ of $T$ and $-T$ is identical);

- $\quad \operatorname{ord}(f, \zeta)$ is the usual order of $f$ at $\zeta$ if $\zeta$ is not a cusp;

- if $\zeta$ is a cusp, $\zeta=T \infty$ where $T=\left(\begin{array}{ll}a & b \\ c & d\end{array}\right) \in \mathrm{SL}_{2}(\mathbb{Z})$, then $\operatorname{ord}(f, \zeta)$ is defined as the order of the series expansion of $(c \tau+d)^{-k} f(T(\tau))$ in $\widetilde{q}=\exp \left(2 i \pi \tau / n_{T}\right)$ (as a Laurent series in $\widetilde{q}$ ), where $k$ is the weight of $f$ and $n_{T}$ is the least positive integer such that $\left(\begin{array}{ll}1 & 1 \\ 0 & 1\end{array}\right)^{n_{T}} \in T \Gamma T^{-1}$;

- $\quad \operatorname{stab}_{\zeta}(\widehat{\Gamma})$ is the subgroup of $\widehat{\Gamma}$ consisting of the elements fixing $\zeta$.

The stabiliser $\operatorname{stab}_{i} \widehat{\Gamma}$ of $i$ in $\widehat{\Gamma}$ can be 2 or 1 depending on whether or not $V=\left(\begin{array}{cc}0 & -1 \\ 1 & 0\end{array}\right)$ or $-V$ is in $\Gamma$. The stabiliser $\operatorname{stab}_{\rho} \widehat{\Gamma}$ of $\rho=\exp (2 i \pi / 3)$ in $\widehat{\Gamma}$ can be 3 or 1 depending on whether or not $P=\left(\begin{array}{cc}0 & -1 \\ 1 & 1\end{array}\right)$ or $-P$ is in $\Gamma$. All other stabilisers consist only of one element.

Below we shall make frequent use of the following special functions. The Eisenstein series (in Ramanujan's notation) $Q(q)$ and $R(q)$ are defined by

$$
Q(q)=1+240 \sum_{n=1}^{\infty} \sigma_{3}(n) q^{n} \quad \text { and } \quad R(q)=1-504 \sum_{n=1}^{\infty} \sigma_{5}(n) q^{n}
$$


and $\sigma_{k}(n)=\sum_{d \mid n} d^{k}$. The Eisenstein series $E_{4}(\tau):=Q(\exp (2 i \pi \tau))$ is a modular form of weight 4 for the full modular group $\mathrm{SL}_{2}(\mathbb{Z})$, while $E_{6}(\tau):=R(\exp (2 i \pi \tau))$ is a modular form of weight 6 for the same group. It is well known (see [33, p. 143]) that the zeros of $Q(q)$ are of the form $q=\exp (2 i \pi \tau)$, where $\tau$ runs through the elements of the orbit of $\rho$ under $\mathrm{SL}_{2}(\mathbb{Z})$. All of these are simple zeros. Similarly (see [33, p. 143]), $R(q)$ has only simple zeros which are of the form $q=\exp (2 i \pi \tau)$, where $\tau$ runs through the elements of the orbit of $i$ under $\mathrm{SL}_{2}(\mathbb{Z})$. The unit circle is a natural boundary for both $Q(q)$ and $R(q)$.

The Dedekind-Klein j-invariant is given by

$$
j(\tau)=1728 \frac{E_{4}^{3}(\tau)}{E_{4}^{3}(\tau)-E_{6}^{2}(\tau)} .
$$

We shall rather use the variant

$$
J(q)=\frac{Q^{3}(q)}{Q^{3}(q)-R^{2}(q)},
$$

so that $j(\tau)=1728 J(\exp (2 i \pi \tau))$. The function $j(\tau)$ is a modular form of weight 0 for $\mathrm{SL}_{2}(\mathbb{Z})$. Further, $J(q)$ is meromorphic in the unit disk with a single pole at $q=0$, which is simple, and the unit circle is a natural boundary.

The Dedekind $\eta$-function is defined by

$$
\eta(\tau)=\exp (i \pi \tau / 12) \prod_{n=1}^{\infty}(1-\exp (2 i \pi n \tau)) .
$$

We shall rather use the variant

$$
H(q)=q^{1 / 24} \prod_{n=1}^{\infty}\left(1-q^{n}\right)
$$

The function $\eta^{24}(\tau)$ can be expressed in terms of Eisenstein series in the form

$$
\eta^{24}(\tau)=E_{4}^{3}(\tau)-E_{6}^{2}(\tau)
$$

and it is therefore a modular form of weight 12 for $\mathrm{SL}_{2}(\mathbb{Z})$.

Two of Jacobi's theta functions will sometimes appear: the function $\theta_{2}(q)$ defined by

$$
\theta_{2}(q)=\sum_{n=-\infty}^{\infty} q^{(n+1 / 2)^{2}}=2 q^{1 / 4} \prod_{j=1}^{\infty}\left(1-q^{2 j}\right)\left(1+q^{2 j}\right)^{2}
$$

(the equality of the two expressions above follows from Jacobi's triple product identity; see [3, Theorem 10.4.1]), and the function $\theta_{3}(q)$ defined by

$$
\theta_{3}(q)=\sum_{n=-\infty}^{\infty} q^{n^{2}}=\prod_{j=1}^{\infty}\left(1-q^{2 j}\right)\left(1+q^{2 j-1}\right)^{2}
$$

(again with Jacobi's triple product identity explaining the equality). 
We shall also frequently use the fact that, if $f(\tau)$ is a modular form of weight $k$ for $\mathrm{SL}_{2}(\mathbb{Z})$, then $f(N \tau)$ is a modular form of weight $k$ for $\Gamma_{0}(N)$, where $\Gamma_{0}(N)$ is the subgroup of $\mathrm{SL}_{2}(\mathbb{Z})$ consisting of all matrices $\left(\begin{array}{ll}a & b \\ c & d\end{array}\right)$ with $c \equiv 0 \bmod N$. Furthermore, $\left|S \widehat{\mathrm{S}_{2}(\mathbb{Z}) / \widehat{\Gamma}_{0}}(2)\right|=3$ and $\left|\mathrm{SL}_{2}(\mathbb{Z}) / \widehat{\Gamma}_{0}(3)\right|=4$ (see [31, Equation (1.4.23)]).

We are now ready to discuss all cases in which $\Phi=1,2,3$.

9.1. $\Phi=1$. There is only a single example in which $\Phi=1$, namely if $\mathbf{N}=(2)$. In that case (see (1.4)), $\mathbf{q}_{(2)}(z)=(1-\sqrt{1-4 z})^{2} /(4 z)$ and $\mathbf{z}_{(2)}(q)=q /(1+q)^{2}$. Hence, $\mathbf{q}_{(2)}(z)$ has exactly one singularity at $z=1 / 4$, which is of square root type, and the radius of convergence is $1 / 4=1 / C$. Moreover, $\mathbf{z}_{(2)}(q)=q /(1+q)^{2}$ has exactly one singularity at $q=-1$. Its radius of convergence is $1=\exp (-\pi \cot (\pi / 2))$.

9.2. $\Phi=2$. In this case, according to Theorem 1.2(ii) and the remark after the statement of the theorem, the point $z=1 / C$ is always a singularity of $\mathbf{q}_{\mathbf{N}}(z)$, with $\mathbf{q}_{\mathbf{N}}(1 / C)=1$. Moreover, because of (4.7), this singularity is of ' $1 /$ log-type'. Consequently, by Lemma 4.6, the mirror map $\mathbf{z}_{\mathbf{N}}(q)$ has an essential singularity at $q=\mathbf{q}_{\mathbf{N}}(1 / C)=1$.

Case $\mathbf{N}=$ (3). Here,

$$
\mathbf{F}_{(3)}(z)=\sum_{n=0}^{\infty} \frac{(3 n) !}{n !^{3}} z^{n}={ }_{2} F_{1}\left[\begin{array}{c}
1 / 3,2 / 3 \\
1
\end{array} 27 z\right] .
$$

It is well known (see, for example, [40, Equation (23)]) that

$$
\mathbf{z}_{(3)}(q)=\left(Q_{3}^{3 / 2}(q)-R_{3}(q)\right) /\left(54 Q_{3}^{3 / 2}(q)\right),
$$

where $Q_{3}(q)=\left(Q(q)+9 Q\left(q^{3}\right)\right) / 10$ and $R_{3}(q)=\left(R(q)+27 R\left(q^{3}\right)\right) / 28$. An alternative expression (see [9, Theorem 2.3]) is given by

$$
\begin{aligned}
\mathbf{z}_{(3)}(q) & =\frac{\left(\theta_{2}\left(q^{1 / 3}\right) \theta_{2}(q)+\theta_{3}\left(q^{1 / 3}\right) \theta_{3}(q)-\theta_{2}(q) \theta_{2}\left(q^{3}\right)-\theta_{3}(q) \theta_{3}\left(q^{3}\right)\right)^{3}}{216\left(\theta_{2}(q) \theta_{2}\left(q^{3}\right)+\theta_{3}(q) \theta_{3}\left(q^{3}\right)\right)^{3}} \\
& =\frac{q}{27 q+\prod_{n=1}^{\infty} \frac{\left(1-q^{n}\right)^{12}}{\left(1-q^{3 n}\right)^{12}}}=\frac{1}{27+\frac{H^{12}(q)}{H^{12}\left(q^{3}\right)}} .
\end{aligned}
$$

The second equality can be easily derived using standard reasoning that involves an estimation of the orders of both expressions at the cusps of a fundamental region of $\Gamma_{0}(12)$, application of the valence formula (9.2), and a verification that sufficiently many coefficients in the power series expansion in $q$ agree; see [15, Sections 3 and 4] for a detailed description of this kind of argument.

Again alternatively, $\mathbf{z}_{(3)}(q)$ is solution of the equation (see [24, Section 5.1, p. 176])

$$
1728 J(q)=\frac{\left(1+216 \mathbf{z}_{(3)}(q)\right)^{3}}{\mathbf{z}_{(3)}(q)\left(1-27 \mathbf{z}_{(3)}(q)\right)^{3}} .
$$

By Lemma 4.1 (with $M=3, L=3, R=2 / 3$ ) and Lemma 4.7, we know that $\mathbf{z}_{(3)}(q)$ has a pole of order three at $q=-\exp (-\pi / \sqrt{3})$. (This corrects the statement in the paragraph 
containing (3) in [38].) Hence, by (9.4), $q=-\exp (-\pi / \sqrt{3})$ must be a zero of $Q_{3}(q)$ of order at least two. Since the function $E_{4}(\tau)$ is a modular form of weight 4 for $\mathrm{SL}_{2}(\mathbb{Z})$, the function $\widetilde{Q}_{3}(\tau):=Q_{3}(\exp (2 i \pi \tau))$ is a modular form of weight 4 for $\Gamma_{0}(3)$. It is easy to see that the orbit of $\rho$ under $\mathrm{SL}_{2}(\mathbb{Z})$ splits under the action of $\Gamma_{0}(3)$ into three orbits: one orbit containing $\rho$, another orbit containing $\rho+1=\frac{1}{2}(1+i \sqrt{3})$, and another orbit containing $\rho_{1}=\frac{1}{2}(1+i / \sqrt{3})$. The reader should note that $\exp \left(2 i \pi \rho_{1}\right)=-\exp (-\pi / \sqrt{3})$. If we now apply the valence formula (9.2) to $\widetilde{Q}_{3}(\tau)$, then, since $\left|\operatorname{stab}_{\rho_{1}}\left(\widehat{\Gamma}_{0}(3)\right)\right|=3$, while $\left|\operatorname{stab}_{\rho}\left(\widehat{\Gamma}_{0}(3)\right)\right|=1$ and $\left|\operatorname{stab}_{i}\left(\widehat{\Gamma}_{0}(3)\right)\right|=1$, we obtain

$$
\frac{1}{3} \operatorname{ord}\left(\widetilde{Q}_{3}, \rho_{1}\right)+\sum_{\zeta \in \mathbb{F} \backslash\left\{\rho_{1}\right\}} \operatorname{ord}\left(\widetilde{Q}_{3}, \zeta\right)=\frac{4}{3} .
$$

We know that $\widetilde{Q}_{3}(\tau)$ is analytic in the upper half plane, that $\operatorname{ord}\left(\widetilde{Q}_{3}, \zeta\right)=0$ for all cusps $\zeta$, and that $\operatorname{ord}\left(\widetilde{Q}_{3}, \rho_{1}\right) \geq 2$. Therefore, if the above equation is to hold then necessarily ord $\left(\widetilde{Q}_{3}, \rho_{1}\right)=4$, and all other orders must be zero. (As a by-product, we see that $R_{3}(q)$ has a zero of order three at $q=-\exp (-\pi / \sqrt{3})$. We remark that the valence formula applied to $R_{3}(q)$ implies that there exists another, simple zero in a fundamental region of $\widehat{\Gamma}_{0}(3)$. We claim that this is $q=\exp (-2 \pi \sqrt{3})$ and all elements in its orbit. To see this, one first observes that (9.3) and the previously quoted fact that $Q(-\exp (-\pi \sqrt{3}))=0$ imply that $J(-\exp (-\pi \sqrt{3}))=0$. Combining this with the modular equation of level 2 , which provides a polynomial relation between $J(q)$ and $J\left(q^{2}\right)$ (see the expression for $H_{2}(x, y)$ in [18, p. 192]), we infer that $J(\exp (-2 \pi \sqrt{3}))=125 / 4$. If this is substituted in (9.5), one obtains four possible values for $\mathbf{z}_{(3)}(\exp (-2 \pi \sqrt{3}))$, one of which is $1 / 54$. By a numerical calculation, the other three values can be ruled out. Finally, by inserting $\mathbf{z}_{(3)}(\exp (-2 \pi \sqrt{3}))=1 / 54$ in $(9.4)$, we see that $R(\exp (-2 \pi \sqrt{3}))=0$, as claimed.)

In summary, the above arguments prove that $\mathbf{z}_{(3)}(q)$ is meromorphic on the unit disk $|q|<1$ with poles of third order at all points $\exp (2 i \pi \tau)$, where $\tau$ is an element of the orbit of $\rho_{1}$ under $\Gamma_{0}(3)$. It is not difficult to see that $\exp \left(2 i \pi \rho_{1}\right)=-\exp (-\pi / \sqrt{3})$ is the element of smallest modulus in this set, and that the other elements become dense near the boundary $|q|=1$. In particular, the Taylor expansion of $\mathbf{z}_{(3)}(q)$ has radius of convergence $\exp (-\pi / \sqrt{3})=\exp (-\pi \cot (\pi / 3))=0.163033 \ldots$, and $\mathbf{z}_{(3)}(q)$ has $|q|=1$ as natural boundary.

Case $\mathbf{N}=(4)$. Here,

$$
\mathbf{F}_{(4)}(z)=\sum_{n=0}^{\infty} \frac{(4 n) !}{(2 n) ! n !^{2}} z^{n}={ }_{2} F_{1}\left[\begin{array}{c}
1 / 4,3 / 4 \\
1
\end{array} ; 64 z\right] .
$$

It is known (see [9, Theorem 2.6]) that

$$
\mathbf{z}_{(4)}(q)=\frac{\theta_{2}^{4}(q) \theta_{3}^{4}(q)}{16\left(\theta_{2}^{4}(q)+\theta_{3}^{4}(q)\right)^{2}}=\frac{q}{64 q+\prod_{n=1}^{\infty} \frac{\left(1-q^{n}\right)^{24}}{\left(1-q^{2 n}\right)^{24}}}=\frac{1}{64+\frac{H^{24}(q)}{H^{24}\left(q^{2}\right)}} .
$$

(The second equality can again be proven by arguments as in [15, Sections 3 and 4].) 
Again alternatively, it is well known that $\mathbf{z}_{(4)}(q)$ is solution of the equation (see [24, Section 5.1, p. 176])

$$
1728 J(q)=\frac{\left(1+192 \mathbf{z}_{(4)}(q)\right)^{3}}{\mathbf{z}_{(4)}(q)\left(1-64 \mathbf{z}_{(4)}(q)\right)^{2}} .
$$

By Lemma 4.1 (with $M=4, L=4, R=3 / 4$ ) and Lemma 4.7, $\mathbf{z}_{(4)}(q)$ has a pole of order two at $q=-\exp (-\pi)$. Hence, by (9.6), $q=-\exp (-\pi)$ must be a zero of order two of $64+H^{24}(q) / H^{24}\left(q^{2}\right)$. The function

$$
f(\tau):=64+\frac{H^{24}(\exp (2 i \pi \tau))}{H^{24}(\exp (4 i \pi \tau))}
$$

is a modular form of weight 0 for $\Gamma_{0}(2)$. It is easy to see that the orbit of $i$ under $\mathrm{SL}_{2}(\mathbb{Z})$ splits under the action of $\Gamma_{0}(2)$ into two orbits: one orbit containing $i$ and another orbit containing $\delta_{1}=\frac{1}{2}(i-1)$. The reader should note that $\exp \left(2 i \pi \delta_{1}\right)=-\exp (-\pi)$. If we now apply the valence formula (9.2) to $f(\tau)$, then, since $\left|\operatorname{stab}_{\delta_{1}}\left(\widehat{\Gamma}_{0}(2)\right)\right|=2$, while $\left|\operatorname{stab}_{i}\left(\widehat{\Gamma}_{0}(2)\right)\right|=1, \operatorname{ord}_{\infty}(f)=-1, \operatorname{ord}_{V \infty}(f)=\operatorname{ord}_{0}(f)=0$, and $\left|\operatorname{stab}_{i}\left(\widehat{\Gamma}_{0}(2)\right)\right|=1$, we obtain

$$
-1+\frac{1}{2} \operatorname{ord}\left(f, \delta_{1}\right)+\sum_{\zeta \in \mathbb{F} \backslash\left\{\delta_{1}, \infty, V \infty\right\}} \operatorname{ord}(f, \zeta)=0 .
$$

We know that $f$ is analytic in the upper half plane and that $\operatorname{ord}\left(f, \delta_{1}\right)=2$. Therefore, if the above equation is to hold then necessarily all other orders must be zero.

In summary, the above arguments prove that $\mathbf{z}_{(4)}(q)$ is meromorphic on the unit disk $|q|<1$ with poles of second order at all points $\exp (2 i \pi \tau)$, where $\tau$ is an element of the orbit of $\delta_{1}$ under $\Gamma_{0}(2)$. It is not difficult to prove that $\exp \left(2 i \pi \delta_{1}\right)=-\exp (-\pi)$ is the element of smallest modulus in this set, and that the other elements become dense near the boundary $|q|=1$. In particular, the Taylor expansion of $\mathbf{z}_{(4)}(q)$ has radius of convergence $\exp (-\pi)=\exp (-\pi \cot (\pi / 4))=0.0432139 \ldots$, and $\mathbf{z}_{(4)}(q)$ has $|q|=1$ as natural boundary.

Case $\mathbf{N}=(6)$. Here,

$$
\mathbf{F}_{(6)}(z)=\sum_{n=0}^{\infty} \frac{(6 n) !}{(3 n) !(2 n) ! n !} z^{n}={ }_{2} F_{1}\left[\begin{array}{c}
1 / 6,5 / 6 \\
1
\end{array} 432 z\right] .
$$

It is well known that $\mathbf{z}_{(6)}(q)$ is solution of the equation (see [24, Section 5.1, p. 176])

$$
1728 J(q)=\frac{1}{\mathbf{z}_{(6)}(q)\left(1-432 \mathbf{z}_{(6)}(q)\right)} .
$$

By Lemma 4.1 (with $M=6, L=6, R=5 / 6$ ) and Lemma 4.7, we know that $\mathbf{z}_{(6)}(q)$ has an algebraic branch point with exponent $-3 / 2$ at $q=-\exp (-\pi \sqrt{3})$. More precisely, since, by solving (9.7),

$$
\mathbf{z}_{(6)}(q)=\frac{1}{864}\left(1-\sqrt{\frac{J(q)-1}{J(q)}}\right)
$$


the only singularities of $\mathbf{z}_{(6)}(q)$ in the interior of the unit disk can occur at points where $J(q)=0$ or $J(q)=1$. These are points $q$ where $Q(q)=0$ or $R(q)=0$. The corresponding values of $q$ are $q=\exp (2 i \pi \tau)$, where $\tau$ runs through the elements of the orbit of $\rho$ or of $i$ under $\mathrm{SL}_{2}(\mathbb{Z})$. However, since $R(q)$ appears as a square in the definition of $J(q)$, each point $q=\exp (2 i \pi \tau)$, where $\tau$ is in the orbit of $i$, is a zero of even order of $J(q)-1$, whence $\sqrt{J(q)-1}$ is analytic at these points.

In summary, the singularities of $\mathbf{z}_{(6)}(q)$ in the interior of the unit disk are $q=$ $\exp (2 i \pi \tau)$, where $\tau$ runs through the elements of the orbit of $\rho$ under $\mathrm{SL}_{2}(\mathbb{Z})$. Each of them is a branch point. It is not difficult to prove that $\exp (2 i \pi \rho)=-\exp (-\pi \sqrt{3})$ is the element of smallest modulus in this set, and that the other elements become dense near the boundary $|q|=1$. In particular, the Taylor expansion of $\mathbf{z}_{(6)}(q)$ has radius of convergence $\exp (-\pi \sqrt{3})=\exp (-\pi \cot (\pi / 6))=0.00433342 \ldots$, and $\mathbf{z}_{(6)}(q)$ has $|q|=1$ as natural boundary.

Case $\mathbf{N}=(2,2)$. Here,

$$
\mathbf{F}_{(2,2)}(z)=\sum_{n=0}^{\infty} \frac{(2 n) !^{2}}{n !^{4}} z^{n}={ }_{2} F_{1}\left[\begin{array}{c}
1 / 2,1 / 2 \\
1
\end{array} ; 16 z\right]
$$

It is well known (see [9, Theorem 2.2]) that

$$
\mathbf{z}_{(2,2)}(q)=\frac{\theta_{2}^{4}(q)}{16 \theta_{3}^{4}(q)}=q \prod_{n=1}^{\infty} \frac{\left(1-q^{4 n}\right)^{8}}{\left(1-(-q)^{n}\right)^{8}}=e^{i \pi / 3} \frac{H^{8}\left(q^{4}\right)}{H^{8}(-q)} .
$$

(In this case, the second equality results upon minor simplification from the product expressions for $\theta_{2}(q)$ and $\theta_{3}(q)$.)

Alternatively, $\mathbf{z}_{(2,2)}(q)$ is solution of the equation (see [24, Section 5.1, p. 176])

$$
1728 J(q)=\frac{\left(1+224 \mathbf{z}_{(2,2)}(q)+256 \mathbf{z}_{(2,2)}^{2}(q)\right)^{3}}{\mathbf{z}_{(2,2)}(q)\left(1-16 \mathbf{z}_{(2,2)}(q)\right)^{4}} .
$$

From (9.8), it is evident that the radius of convergence of $\mathbf{z}_{(2,2)}(q)$ is $1=$ $\exp (-\pi \cot (\pi / 2))$ and that $\mathbf{z}_{(2,2)}(q)$ has $|q|=1$ as natural boundary. We remark that this is consistent with Lemmas 4.1(iii) and 4.7(iii), which imply that $\mathbf{z}_{(2,2)}(q)$ has an essential singularity at $q=-\exp (-\pi \cot (\pi / 2))=-1$.

9.3. $\Phi=3$. In this case, according to Theorem 1.2 (ii), the point $z=1 / C$ is always a singularity of $\mathbf{q}_{\mathbf{N}}(z)$. Furthermore, because of Lemma 4.3(ii) (with $d=0$ ), this singularity is of square root type. Consequently, according to Lemma 4.4 (with $d=0$ ), the mirror map $\mathbf{z}_{\mathbf{N}}(q)$ is analytic at $\mathbf{q}_{\mathbf{N}}(1 / C)$, with $\mathbf{z}_{\mathbf{N}}^{\prime}\left(\mathbf{q}_{\mathbf{N}}(1 / C)\right)=0$.

Case $\mathbf{N}=(2,3)$. Here,

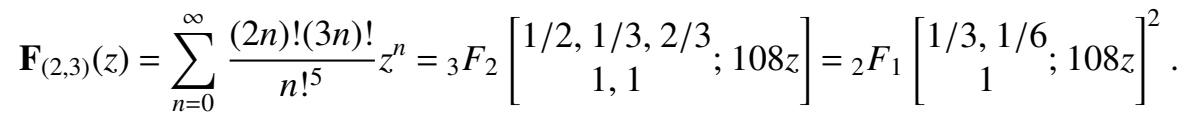


The identity between the hypergeometric series is a special case of Clausen's formula (see [34, Equation (2.5.7)])

$$
{ }_{3} F_{2}\left[\begin{array}{c}
2 a, 2 b, a+b \\
2 a+2 b, a+b+\frac{1}{2} ; z
\end{array}\right]={ }_{2} F_{1}\left[\begin{array}{c}
a, b \\
a+b+\frac{1}{2} ; z
\end{array}\right]^{2} .
$$

It is well known (see, for example, [40, Equation (23)]) that

$$
\mathbf{z}_{(2,3)}(q)=\left(Q_{3}^{3}(q)-R_{3}^{2}(q)\right) /\left(108 Q_{3}^{3}(q)\right)
$$

where $Q_{3}(q)$ and $R_{3}(q)$ are defined as in the case where $\mathbf{N}=(3)$. From the considerations concerning the zeros of $Q_{3}(q)$ and $R_{3}(q)$ in the case where $\mathbf{N}=(3)$, we conclude that $\mathbf{z}_{(2,3)}(q)$ has poles of order six at all points $\exp (2 i \pi \tau)$, where $\tau$ is an element of the orbit of $\rho_{1}=\frac{1}{2}(1+i / \sqrt{3})$ under $\Gamma_{0}(3)$. The point $\exp \left(2 i \pi \rho_{1}\right)=$ $-\exp (-\pi / \sqrt{3})$ is the element of smallest modulus in this set, and the other elements become dense near the boundary $|q|=1$. In particular, the Taylor expansion of $\mathbf{z}_{(2,3)}(q)$ has radius of convergence $\exp (-\pi / \sqrt{3})=\exp (-\pi \cot (\pi / 3))=0.163033 \ldots$, and $\mathbf{z}_{(2,3)}(q)$ has $|q|=1$ as natural boundary.

Case $\mathbf{N}=(2,4)$. Here,

$$
\mathbf{F}_{(2,4)}(z)=\sum_{n=0}^{\infty} \frac{(4 n) !}{n !^{4}} z^{n}={ }_{3} F_{2}\left[\begin{array}{c}
1 / 2,1 / 4,3 / 4 \\
1,1
\end{array} ; 256 z\right]={ }_{2} F_{1}\left[\begin{array}{c}
1 / 8,3 / 8 \\
1
\end{array} 256 z\right]^{2}
$$

again with Clausen's formula (9.9) explaining the identity between the hypergeometric series. It is well known (see, for example, [40, Equation (23)]) that

$$
\mathbf{z}_{(2,4)}(q)=\left(Q_{2}^{3}(q)-R_{2}^{2}(q)\right) /\left(256 Q_{2}^{3}(q)\right)
$$

where $Q_{2}(q)=\left(Q(q)+4 Q\left(q^{2}\right)\right) / 5$ and $R_{2}(q)=\left(R(q)+8 R\left(q^{2}\right)\right) / 9$. An alternative expression can be found if one observes that, by the quadratic transformation formula (see [6, Example 4(iii), p. 97])

$$
{ }_{2} F_{1}\left[\begin{array}{c}
a, b \\
\frac{1}{2}+a+b
\end{array} ; z\right]={ }_{2} F_{1}\left[\begin{array}{c}
2 a, 2 b \\
\frac{1}{2}+a+b
\end{array} ; \frac{1-\sqrt{1-z}}{2}\right],
$$

we have

$$
{ }_{2} F_{1}\left[\begin{array}{c}
1 / 8,3 / 8 \\
1
\end{array} 256 z\right]={ }_{2} F_{1}\left[\begin{array}{c}
1 / 4,3 / 4 \\
1
\end{array} ; \frac{1-\sqrt{1-256 z}}{2}\right],
$$

which enables us to identify $\frac{1}{2}\left(1-\sqrt{1-256 \mathbf{z}_{(2,4)}(q)}\right)$ with $64 \mathbf{z}_{(4)}(q)$, or, explicitly,

$$
\mathbf{z}_{(2,4)}(q)=\mathbf{z}_{(4)}(q)-64 \mathbf{z}_{(4)}^{2}(q) \text {. }
$$

By Lemma 4.1 (with $M=4, L=4, R=1 / 2$ ) and Lemma 4.7, we know that $\mathbf{z}_{(2,4)}(q)$ has a pole of order four at $q=-\exp (-\pi)$. We can now either use expression (9.10) 
in combination with arguments as in the case where $\mathbf{N}=(4)$, or (9.12) and similar arguments, to conclude that $\mathbf{z}_{(2,4)}(q)$ is meromorphic on the unit disk $|q|<1$ with poles of fourth order at all points $\exp (2 i \pi \tau)$, where $\tau$ is an element of the orbit of $\delta_{1}=\frac{1}{2}(i-1)$ under $\Gamma_{0}(2)$. The point $\exp \left(2 i \pi \delta_{1}\right)=-\exp (-\pi)$ is the element of smallest modulus in this set, and the other elements become dense near the boundary $|q|=1$. In particular, the Taylor expansion of $\mathbf{z}_{(2,4)}(q)$ has radius of convergence $\exp (-\pi)=$ $\exp (-\pi \cot (\pi / 4))=0.0432139 \ldots$, and $\mathbf{z}_{(2,4)}(q)$ has $|q|=1$ as natural boundary.

We remark that, as a by-product, we obtain that $Q_{2}(q)$ has a zero of order two and $R_{2}(q)$ has a simple zero at $q=-\exp (-\pi)$.

Case $\mathbf{N}=(2,6)$. Here,

$$
\mathbf{F}_{(2,6)}(z)=\sum_{n=0}^{\infty} \frac{(6 n) !}{(3 n) ! n !^{3}} z^{n}={ }_{3} F_{2}\left[\begin{array}{c}
1 / 2,1 / 6,5 / 6 \\
1,1
\end{array} ; 1728 z\right]={ }_{2} F_{1}\left[\begin{array}{c}
1 / 12,5 / 12 ; 1728 z \\
1
\end{array}\right]^{2},
$$

again with Clausen's formula (9.9) behind the identity between the hypergeometric series. It is well known (see [25, Section 2]) that $\mathbf{z}_{(2,6)}(q)=1 /(1728 J(q))$. By the considerations in the case where $\mathbf{N}=(6)$, we conclude that $\mathbf{z}_{(2,6)}(q)$ has a pole of order three at all points $q=\exp (2 i \pi \tau)$, where $\tau$ runs through the elements of the orbit of $\rho$ under $\mathrm{SL}_{2}(\mathbb{Z})$. The point $\exp (2 i \pi \rho)=-\exp (-\pi \sqrt{3})$ is the element of smallest modulus in this set, and the other elements become dense near the boundary $|q|=1$. In particular, the Taylor expansion of $\mathbf{z}_{(2,6)}(q)$ has radius of convergence $\exp (-\pi \sqrt{3})=$ $\exp (-\pi \cot (\pi / 6))=0.00433342 \ldots$, and $\mathbf{z}_{(2,6)}(q)$ has $|q|=1$ as natural boundary.

Case $\mathbf{N}=(2,2,2)$. Here,

$$
\mathbf{F}_{(2,2,2)}(z)=\sum_{n=0}^{\infty} \frac{(2 n) !^{3}}{n !^{6}} z^{n}={ }_{3} F_{2}\left[\begin{array}{c}
1 / 2,1 / 2,1 / 2 \\
1,1
\end{array}{ }^{2} 64 z\right]={ }_{2} F_{1}\left[\begin{array}{c}
1 / 4,1 / 4 \\
1
\end{array}{ }^{2} 64 z\right]^{2},
$$

again with Clausen's formula (9.9) behind the identity between the hypergeometric series. By applying (9.11), we can relate the ${ }_{2} F_{1}$-series to the ${ }_{2} F_{1}$-series from the case where $\mathbf{N}=(2,2)$ :

$$
{ }_{2} F_{1}\left[\begin{array}{c}
1 / 4,1 / 4 \\
1
\end{array} ; 64 z\right]={ }_{2} F_{1}\left[\begin{array}{c}
1 / 2,1 / 2 ; \frac{1-\sqrt{1-64 z}}{2} \\
1
\end{array}\right],
$$

which enables us to identify $\frac{1}{2}\left(1-\sqrt{1-64 \mathbf{z}_{(2,2,2)}(q)}\right)$ with $16 \mathbf{z}_{(2,2)}(q)$, or, explicitly,

$$
\mathbf{z}_{(2,2,2)}(q)=\mathbf{z}_{(2,2)}(q)-16 \mathbf{z}_{(2,2)}^{2}(q) .
$$

The expression on the right-hand side of (9.13) can be rewritten in the form

$$
\mathbf{z}_{(2,2,2)}(q)=q \prod_{n=1}^{\infty} \frac{\left(1-q^{2 n}\right)^{24}}{\left(1-(-q)^{n}\right)^{24}}=-\frac{H^{24}\left(q^{2}\right)}{H^{24}(-q)} .
$$

(This follows again by arguments as in [15, Sections 3 and 4].) From (9.14), it is evident that the radius of convergence of $\mathbf{z}_{(2,2,2)}(q)$ is $1=\exp (-\pi \cot (\pi / 2))$, and 
that $\mathbf{z}_{(2,2,2)}(q)$ has $|q|=1$ as natural boundary. We remark that this is consistent with Lemmas 4.1(iii) and 4.7(iii), which imply that $\mathbf{z}_{(2,2,2)}(q)$ has an essential singularity at $q=-\exp (-\pi \cot (\pi / 2))=-1$.

\section{Proof of Proposition 1.6}

By Theorem 1.2(i), the radius of convergence of $\mathbf{q}_{\mathbf{N}}(z)$ is $1 / C$. Moreover, by Theorem 1.1, the Taylor coefficients of $\mathbf{q}_{\mathbf{N}}(z)$ at 0 are all positive. Hence, $\mathbf{q}_{\mathbf{N}}^{\prime}(z)>$ 0 for all $z$ in the segment $[0,1 / C)$. By the inverse function theorem, $\mathbf{q}_{\mathbf{N}}(z)$ has a compositional inverse in a domain containing $[0,1 / C)$. This implies the first assertion.

The additional assertion on the analytic nature of $\mathbf{z}_{\mathbf{N}}(q)$ around $\mathbf{q}_{\mathbf{N}}(1 / C)$ results from Lemma 4.3(ii), (iii). Strictly speaking, the lemma makes no assertion about the angle (depending on $\varepsilon$ ) of the slit neighbourhood at $1 / C$. However, it is not very difficult to see that the assertion that there exists such a slit neighbourhood with an arbitrary $\varepsilon>0$ can be derived by an appropriate refinement of the proof of Lemma 4.3.

The argument for the assertion on the analytic nature of $\mathbf{z}_{\mathbf{N}}(q)$ around the point $-\exp (-\pi \cot (\pi / M))$ given in the second paragraph after Conjecture 1.10 is similar. It is based on the singular expansion of $\mathbf{q}_{\mathbf{N}}(z)$ at $z=\infty$ given in Lemma 4.1 and its consequence for the singular expansion of $\mathbf{z}_{\mathbf{N}}(q)$ at $-\exp (-\pi \cot (\pi / M))$ given in Lemma 4.7.

\section{What do we need to prove Conjecture 1.8 ?}

By the Lagrange inversion formula (see [17, Theorem 1.9b with $R(x)=x]$ ), we can express the $m$ th Taylor coefficient of $\mathbf{z}_{\mathbf{N}}(q)$ in the form

$$
\frac{1}{2 i \pi m} \int_{\mathcal{D}} \frac{d z}{\mathbf{q}_{\mathbf{N}}^{m}(z)},
$$

where $\mathcal{D}$ is a sufficiently small contour that encircles the origin once in positive (that is, counter-clockwise) direction. We now deform $\mathcal{D}$ to a contour $\mathscr{C}$ consisting of four parts,

$$
\mathscr{C}=\mathscr{C}_{1} \cup \mathscr{C}_{+} \cup \mathscr{C}_{-} \cup \mathscr{C}_{0}
$$

where:

(1) $\mathscr{C}_{1}$ is a (small) piece of length $\ell$, say, that passes through $1 / C$;

(2) $\mathscr{C}_{\circ}$ is a segment $\left\{\operatorname{Re}^{i \theta}: \delta \leq \theta \leq 2 \pi-\delta\right\}$ of a (large) circle of radius $R$, for some $\delta>0$

(3) $\mathscr{C}_{+}$is a path that connects the ends of $\mathscr{C}_{1}$ and $\mathscr{C}_{\circ}$ with positive real parts;

(4) $\mathscr{C}_{-}$is a path that connects the ends of $\mathscr{C}_{1}$ and $\mathscr{C}_{0}$ with negative real parts;

(5) $\left|\mathbf{q}_{\mathbf{N}}(z)\right| \geq \mathbf{q}_{\mathbf{N}}(1 / C)$ for all $z \in \mathscr{C}$,

if such a contour exists (see Figure 2 for a symbolic illustration). There is no problem with the existence of $\mathscr{C}_{1}$ since Lemma 4.3(ii), (iii) says that the coefficient of $1-C z$ in 


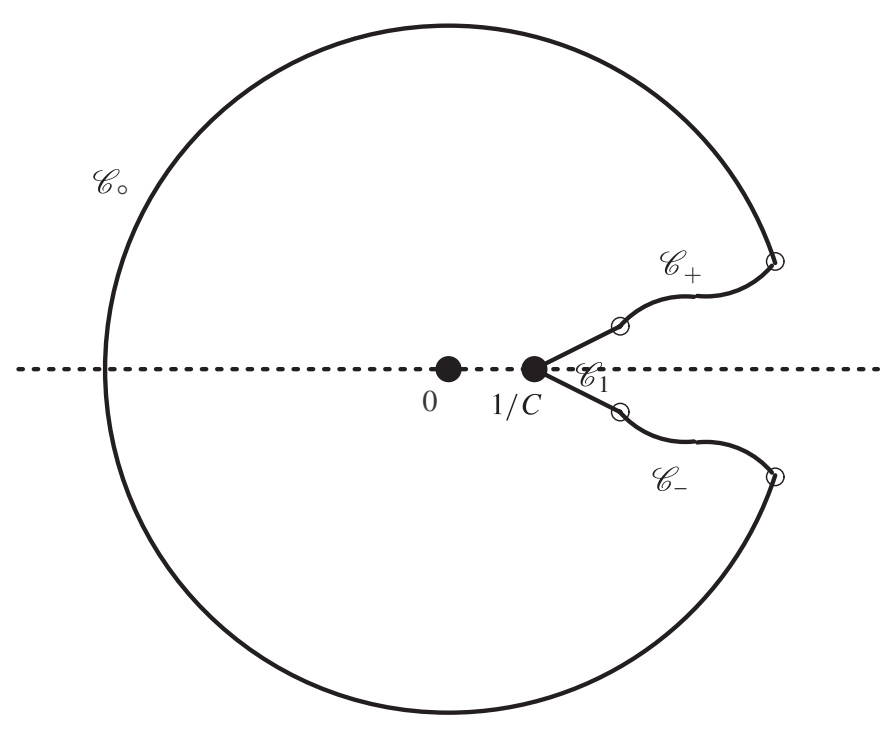

FIGURE 2. The contour $\mathscr{C}$.

the singular expansion of $\mathbf{q}_{\mathbf{N}}(z)$ about $z=1 / C$ must be negative, nor is there with the existence of $\mathscr{C}_{\circ}$, the latter due to Theorem 1.3 and Lemma 8.1. The problem here is the question of existence of a suitable path $\mathscr{C}_{+}$connecting a point in the neighbourhood of $\mathbf{q}_{\mathbf{N}}(1 / C)$ with a point 'far out' (these points being indicated by circles in Figure 2) so that, along the path, all values of $\mathbf{q}_{\mathbf{N}}(z)$ are larger in modulus than $\mathbf{q}_{\mathbf{N}}(1 / C)$. There cannot be any doubt that, if $\Phi \geq 4$, such a path exists, but, unfortunately, we have not been able to establish this. It is easy to see that (by exploiting that $\mathbf{q}_{\mathbf{N}}(\bar{z})=\overline{\mathbf{q}_{\mathbf{N}}(z)}$ ), once we know that a suitable path $C_{+}$exists, there is also a suitable path $C_{-}$.

Let us continue under the assumption that paths $C_{+}$and $C_{-}$as described above exist. Then we may estimate

$$
\left|\frac{1}{2 i \pi m} \int_{\mathcal{D}} \frac{d z}{\mathbf{q}_{\mathbf{N}}^{m}(z)}\right|=\left|\frac{1}{2 i \pi m} \int_{\mathscr{C}} \frac{d z}{\mathbf{q}_{\mathbf{N}}^{m}(z)}\right| \leq \frac{b}{m \times \mathbf{q}_{\mathbf{N}}^{m}(1 / C)},
$$

where $b$ is some constant. Since the contour integral (11.1) gives the coefficient of $q^{m}$ in the Taylor expansion of $\mathbf{z}_{\mathbf{N}}(q)$, this implies that the radius of convergence of the series $\mathbf{z}_{\mathbf{N}}(q)$ must be at least $\mathbf{q}_{\mathbf{N}}(1 / C)$. From Theorem 1.4(ii) it then follows that it must be exactly $\mathbf{q}_{\mathbf{N}}(1 / C)$.

Whether or not $\mathbf{q}_{\mathbf{N}}(1 / C)$ is the only point of singularity of $\mathbf{z}_{\mathbf{N}}(q)$ on the boundary of its disk of convergence would have to be decided by an additional argument.

\section{Conjecture 1.9 implies the first assertion in Conjecture 1.8}

Let $\Phi \geq 4$. Conjecture 1.9 says that all but a finite number of coefficients in the Taylor series expansion of $\mathbf{z}_{\mathbf{N}}(q)$ are negative. Let this Taylor series expansion 
be $\sum_{m=0}^{\infty} \mathbf{z}_{m} q^{m}$. A well-known theorem of Pringsheim (see [14, Theorem IV.6]) says that, if a function $f(z)$ can be represented in a neighbourhood of $z=0$ as a power series with nonnegative coefficients with radius of convergence $R$, then $f(z)$ has a singularity at $z=R$. If we apply this theorem to $-\sum_{m=4}^{\infty} \mathrm{z}_{m} q^{m}$, then we conclude that the radius of convergence of $\mathbf{z}_{\mathbf{N}}(q)$ is a positive real number which is at the same time a singularity of $\mathbf{z}_{\mathbf{N}}(q)$. By Proposition 1.6, we know that $\mathbf{z}_{\mathbf{N}}(q)$ is analytic at all points $q \in\left[0, \mathbf{q}_{\mathbf{N}}(1 / C)\right)$. Thus, we know that the radius of convergence of $\mathbf{z}_{\mathbf{N}}(q)$ must be at least $\mathbf{q}_{\mathbf{N}}(1 / C)$. On the other hand, by Theorem 1.4 we know that it cannot be larger, whence the conclusion follows.

\section{Conjecture 1.8 implies a weak version of Conjecture 1.9(ii) and (iii)}

Here we prove that Conjecture 1.8 implies that, if $\Phi_{\mathbf{N}} \geq 4$, almost all coefficients in the Taylor expansion of $\mathbf{z}_{\mathbf{N}}(q)$ at $q=0$ are negative.

Proposition 13.1. Let $N_{1}, N_{2}, \ldots, N_{k}$ be positive integers, all at least 2 , such that $\Phi_{\mathbf{N}} \geq 4$, and assume that Conjecture 1.8 holds. Then there exists an $A_{\mathbf{N}}>0$ such that the coefficient of $q^{m}$ in the Taylor series of $\mathbf{z}_{\mathbf{N}}(q)$ is negative for every $m>A_{\mathbf{N}}$.

Proof. By Lemma 4.3(ii), (iii), we know that $\mathbf{q}_{\mathbf{N}}(z)$ admits a singular expansion at $z=1 / C$ of the form

$$
\begin{aligned}
\mathbf{q}_{\mathbf{N}}(z)=\mathbf{q}_{\mathbf{N}}(1 / C)+\mathrm{q}_{1}(1-C z)+\mathrm{q}_{2}(1-C z)^{2}+\cdots \\
\quad+\mathrm{q}_{d-1}(1-C z)^{d-1}+Q_{d}(1-C z)^{d} \widetilde{L}(z)+O\left((1-C z)^{d}\right),
\end{aligned}
$$

where $d=\lfloor(\Phi-2) / 2\rfloor, \mathrm{q}_{1}<0,(-1)^{d+1} Q_{d}>0$, and

$$
\widetilde{L}(z)= \begin{cases}(1-C z)^{1 / 2} & \text { if } \Phi \text { is odd } \\ -\log (1-C z) & \text { if } \Phi \text { is even }\end{cases}
$$

By Lemmas 4.4 and 4.5 , it follows that $\mathbf{z}_{\mathbf{N}}(q)$ admits a singular expansion at $q=$ $\mathbf{q}_{\mathbf{N}}(1 / C)$ of the form

$$
\begin{aligned}
\mathbf{z}_{\mathbf{N}}(q)=\frac{1}{C} & +\mathrm{z}_{1}\left(q-\mathbf{q}_{\mathbf{N}}(1 / C)\right)+\mathrm{z}_{2}\left(q-\mathbf{q}_{\mathbf{N}}(1 / C)\right)^{2}+\cdots \\
& +\mathrm{z}_{d-1}\left(q-\mathbf{q}_{\mathbf{N}}(1 / C)\right)^{d-1}+Z_{d}\left(q-\mathbf{q}_{\mathbf{N}}(1 / C)\right)^{d} \widehat{L}(q) \\
& +O\left(\left(q-\mathbf{q}_{\mathbf{N}}(1 / C)\right)^{d+1}\right)
\end{aligned}
$$

where $d=\lfloor(\Phi-2) / 2\rfloor, \mathrm{z}_{1}=-1 /\left(C \mathrm{q}_{1}\right), Z_{d}>0$, and

$$
\widehat{L}(z)= \begin{cases}\left(\mathbf{q}_{\mathbf{N}}(1 / C)-q\right)^{1 / 2} & \text { if } \Phi \text { is odd } \\ -\log \left(\mathbf{q}_{\mathbf{N}}(1 / C)-q\right) & \text { if } \Phi \text { is even }\end{cases}
$$

If we now use Conjecture 1.8, then the standard theorems of singularity analysis (see [14, Ch. VI]) imply that the term in $(13.1)$ containing $\widehat{L}(q)$ contributes the main 
term to the asymptotics of the Taylor coefficients of $\mathbf{z}_{\mathbf{N}}(q)$. More precisely, as $m \rightarrow \infty$, the $m$ th Taylor coefficient of $\mathbf{z}_{\mathbf{N}}(q)$ is equal to

$$
-K \frac{1+o(1)}{\mathbf{q}_{\mathbf{N}}^{m}(1 / C) m^{\Phi / 2}},
$$

where $K$ is a positive constant which can be computed explicitly in terms of $Z_{d}, \Phi$. The claim of the proposition is now obvious.

\section{Acknowledgements}

The authors thank Bruce Berndt and Heng-Huat Chan for very helpful correspondence concerning the discussion of modular mirror maps presented here in Section 9.

\section{References}

[1] G. Almkvist and W. Zudilin, 'Differential equations, mirror maps and zeta values', in: Mirror Symmetry V, AMS/IP Studies in Advanced Mathematics, 38 (eds. N. Yui, S.-T. Yau and J. D. Lewis) (American Mathematical Society, Providence, RI; International Press, Somerville, MA, 2007), pp. 481-515.

[2] Y. André, 'G-fonctions et transcendance', J. reine angew. Math. 476 (1996), 95-125.

[3] G. E. Andrews, R. A. Askey and R. Roy, Special Functions, The Encyclopedia of Mathematics and Its Applications, 71 (Cambridge University Press, Cambridge, 1999).

[4] T. Asai, M. Kaneko and H. Ninomiya, 'Zeros of certain modular functions and an application', Comment. Math. Univ. St. Pauli 46 (1997), 93-101.

[5] E. Bach and J. O. Shallit, Algorithmic Number Theory, Vol. 1 (MIT Press, Cambridge, MA, 1996).

[6] W. N. Bailey, Generalized Hypergeometric Series (Cambridge University Press, Cambridge, 1935).

[7] F. Beukers, 'Irrationality proofs using modular forms', in: Journées arithmétiques de Besançon (Besançon, 1985), Astérisque, 147-148 (Soc. Math. France, Paris, 1987), pp. 271-283, 345.

[8] F. Beukers and G. Heckman, 'Monodromy for the hypergeometric function ${ }_{n} F_{n-1}$ ', Invent. Math. 95 (1989), 325-354.

[9] J. M. Borwein and P. B. Borwein, 'A cubic counterpart of Jacobi's identity and the AGM', Trans. Amer. Math. Soc. 323 (1991), 691-701.

[10] P. Candelas, X. de la Ossa, P. Green and L. Parkes, 'A pair of Calabi-Yau manifolds as an exactly soluble superconformal theory', Nucl. Phys. B359 (1991), 21-74.

[11] E. A. Coddington and N. Levinson, Theory of Ordinary Differential Equations (McGraw-Hill, New York, 1955).

[12] N. G. De Bruijn, Asymptotic Methods in Analysis (North-Holland, Amsterdam, 1958).

[13] E. Delaygue, 'Critère pour l'integralité des coefficients de Taylor des applications miroir', J. reine angew. Math. 662 (2012), 205-252.

[14] P. Flajolet and R. Sedgewick, Analytic Combinatorics (Cambridge University Press, Cambridge, 2009).

[15] F. Garvan, 'Shifted and shiftless partition identities II', in: Number Theory for the Millennium II, (eds. M. A. Bennett, B. C. Berndt, N. Boston, H. G. Diamond, A. J. Hildebrand and W. Philipp) (A K Peters, Natick, MA, 2002), pp. 75-92.

[16] F. Hausdorff, 'Momentprobleme für ein endliches Intervall', Math. Z. 16 (1923), 220-248.

[17] P. Henrici, Applied and Computational Complex Analysis, Vol. 1 (Wiley, New York, 1974).

[18] O. Herrmann, 'Über die Berechnung der Fourierkoeffizienten der Funktion $j(\tau)$ ', J. reine angew. Math. 274/275 (1973/74), 187-195.

[19] T. Kaluza, 'Über die Koeffizienten reziproker Potenzreihen', Math. Z. 28 (1928), 161-170.

[20] C. Krattenthaler and T. Rivoal, 'On the integrality of Taylor coefficients of mirror maps', Duke Math. J. 151 (2010), 175-218. 
[21] C. Krattenthaler and T. Rivoal, 'Multivariate $p$-adic formal congruences and integrality of Taylor coefficients of mirror maps', in: Théories Galoisiennes et Arithmétiques des Équations Différentielles, Séminaires et Congrès, 27 (eds. L. Di Vizio and T. Rivoal) (Société Mathématique de France, Paris, 2011), pp. 279-307.

[22] J. Lamperti, 'On the coefficients of reciprocal power series', Amer. Math. Monthly 65 (1958), 90-94.

[23] S.-C. Lau, N. C. Leung and B. Wu, 'Mirror maps equal SYZ maps for toric Calabi-Yau surfaces', Bull. London Math. Soc. 44 (2012), 255-270.

[24] B. H. Lian and S.-T. Yau, 'Arithmetic properties of mirror map and quantum coupling', Comm. Math. Phys. 176 (1996), 163-191.

[25] B. H. Lian and S.-T. Yau, 'Mirror maps, modular relations and hypergeometric series I', appeared as 'Integrality of certain exponential series', in: Lectures in Algebra and Geometry, Proceedings of the International Conference on Algebra and Geometry (Taipei, 1995) (ed. M.-C. Kang) (International Press, Cambridge, MA, 1998), pp. 215-227.

[26] D. R. Morrison, 'Mirror symmetry and rational curves on quintic threefolds: a guide for mathematicians', J. Amer. Math. Soc. 6 (1993), 223-247.

[27] R. Pandharipande, 'Rational curves on hypersurfaces (after A. Givental)', in: Séminaire Bourbaki, Vol. 1997/98, Astérisque, 252 (Soc. Math. France, Paris, 1998), Exp. No. 848, 5, 307-340.

[28] The PARI Group, PARI/GP. http://pari.math.u-bordeaux.fr.

[29] G. Pólya, 'Application of a theorem connected with the problem of moments', Mess. Math. 55 (1926), 189-192.

[30] A. J. van der Poorten, 'A proof that Euler missed: Apéry's proof of the irrationality of $\zeta(3)$ ', Math. Intelligencer 1 (1978/79), 195-203.

[31] R. A. Rankin, Modular Forms and Functions (Cambridge University Press, Cambridge, 1977).

[32] Z. Sasvári, 'An elementary proof of Binet's formula for the gamma function', Amer. Math. Monthly 106 (1999), 156-158.

[33] J.-P. Serre, Cours d'arithmétique (deuxième édition revue et corrigée), Le Mathématicien, 2, (Presses Universitaires de France, Paris, 1977).

[34] L. J. Slater, Generalized Hypergeometric Functions (Cambridge University Press, Cambridge, 1966).

[35] P. F. Stiller, 'Classical automorphic forms and hypergeometric functions', J. Number Theory $\mathbf{2 8}$ (1988), 219-232.

[36] C. Voisin, Mirror Symmetry, SMF/AMS Texts and Monographs, 1 (American Mathematical Society, Providence, RI; Société Mathématique de France, Paris, 1999).

[37] K. J. Wirths, 'Über totalmonotone Zahlenfolgen', Arch. Math. 26 (1975), 508-517.

[38] W. Zudilin, 'Number theory casting a look at the mirror', Preprint, arXiv:math.NT/0008237.

[39] W. Zudilin, 'Integrality of power expansions related to hypergeometric series', Math. Notes 71 (2002), 604-616.

[40] W. Zudilin, 'The hypergeometric equation and Ramanujan functions', Ramanujan J. 7 (2003), $435-447$.

\author{
C. KRATTENTHALER, Fakultät für Mathematik, Universität Wien, \\ Nordbergstraße 15, A-1090 Vienna, Austria \\ e-mail: Christian.Krattenthaler@univie.ac.at
}

\author{
T. RIVOAL, Institut Fourier, CNRS UMR 5582, Université Grenoble 1, \\ 100 rue des Maths, BP 74, 38402 Saint-Martin d'Hères cedex, France \\ and \\ Current address: Institut Camille Jordan, CNRS et Université Lyon 1, \\ 43 boulevard du 11 novembre 1918, 69622 Villeurbanne cedex, France \\ e-mail: rivoal@math.univ-lyon1.fr
}

